\title{
Changes in the X-ray Emission from the Magnetar Candidate 1E 2259+ 586 during its 2002 Outburst
}

\section{Citation}

Woods, P. M., V. M. Kaspi, C. Thompson, F. P. Gavriil, H. L. Marshall, D. Chakrabarty, K. Flanagan, J. Heyl, and L. Hernquist. 2004. "Changes in the X\#Ray Emission from the Magnetar Candidate 1E 2259+586 during Its 2002 Outburst." The Astrophysical Journal 605 (1): 378-99. https:// doi.org/10.1086/382233.

\section{Permanent link}

http://nrs.harvard.edu/urn-3:HUL.InstRepos:41381758

\section{Terms of Use}

This article was downloaded from Harvard University's DASH repository, and is made available under the terms and conditions applicable to Other Posted Material, as set forth at http:// nrs.harvard.edu/urn-3:HUL.InstRepos:dash.current.terms-of-use\#LAA

\section{Share Your Story}

The Harvard community has made this article openly available. Please share how this access benefits you. Submit a story.

Accessibility 


\title{
Changes in the X-ray Emission from the Magnetar Candidate 1E 2259+586 during its 2002 Outburst
}

\author{
P.M. Woods ${ }^{1,2}$, V.M. Kaspi ${ }^{3,4}$, C. Thompson ${ }^{5}$, F.P. Gavriil ${ }^{3}$, H.L. Marshall ${ }^{4}$, D. Chakrabarty ${ }^{4}$, \\ K. Flanagan ${ }^{4}$, J. Heyl ${ }^{6,7}$, and L. Hernquist ${ }^{6}$
}

\begin{abstract}
An outburst of more than 80 individual bursts, similar to those seen from Soft Gamma Repeaters (SGRs), was detected from the Anomalous X-ray Pulsar (AXP) $1 \mathrm{E} 2259+586$ in 2002 June. Coincident with this burst activity were gross changes in the pulsed flux, persistent flux, energy spectrum, pulse profile and spin down of the underlying X-ray source. We present Rossi X-ray Timing Explorer and X-ray Multi-Mirror Mission observations of 1E 2259+586 that show the evolution of the aforementioned source parameters during and following this episode and identify recovery time scales for each. Specifically, we observe an X-ray flux increase (pulsed and phase-averaged) by more than an order of magnitude having two distinct components. The first component is linked to the burst activity and decays within $\sim 2$ days during which the energy spectrum is considerably harder than during the quiescent state of the source. The second component decays over the year following the glitch according to a power law in time with an exponent $-0.22 \pm 0.01$. The pulsed fraction decreased initially to $\sim 15 \%$ RMS, but recovered rapidly to the pre-outburst level of $\sim 23 \%$ within the first three days. The pulse profile changed significantly during the outburst, and recovered almost fully within two months of the outburst. A glitch of size $\Delta \nu_{\max } / \nu=(4.24 \pm 0.11) \times 10^{-6}$ was observed in $1 \mathrm{E} 2259+586$ that preceded the observed burst activity. The glitch could not be well fit with a simple partial exponential recovery. An exponential rise of $\sim 20 \%$ of the frequency jump with a time scale of $\sim 14$ days results in a significantly better fit to the data, however contamination from a systematic drift in the phase of the pulse profile cannot be
\end{abstract}

\footnotetext{
${ }^{1}$ Universities Space Research Association; peter.woods@nsstc.nasa.gov

${ }^{2}$ National Space Science and Technology Center, 320 Sparkman Dr. Huntsville, AL 35805

${ }^{3}$ Department of Physics, Rutherford Physics Building, McGill University, 3600 University St., Montreal, Quebec, H3A 2T8, Canada

${ }^{4}$ Center for Space Research, Massachusetts Institute of Technology, Cambridge, MA 02139

${ }^{5}$ Canadian Institute for Theoretical Astrophysics, 60 St. George Street, Toronto, ON M5S 3H8, Canada

${ }^{6}$ Harvard-Smithsonian Center for Astrophysics, 60 Garden St., Cambridge, MA 02138

${ }^{7}$ Chandra Fellow, Current Address: Department of Physics and Astronomy, 6224 Agricultural Road, Vancouver BC V6T 1Z1, Canada
} 
excluded. A fraction of the glitch $(\sim 19 \%)$ recovered in a quasi-exponential manner having a recovery time scale of $\sim 16$ days. The long-term post-glitch spin-down rate decreased in magnitude relative to the pre-glitch value. The changes in the source properties of $1 \mathrm{E} 2259+586$ during its 2002 outburst are shown to be qualitatively similar to changes seen during/following burst activity in two SGRs, thus further solidifying the common nature of SGRs and AXPs as magnetars. The changes in persistent emission properties of $1 \mathrm{E} 2259+586$ suggest that the star underwent a plastic deformation of the crust that simultaneously impacted the superfluid interior (crustal and possibly core superfluid) and the magnetosphere. Finally, the changes in persistent emission properties coincident with burst activity in 1E 2259+586 enabled us to infer previous burst active episodes from this and other AXPs. The non-detection of these outbursts by all-sky gamma-ray instruments suggests that the number of active magnetar candidates in our Galaxy is larger than previously thought.

Subject headings: stars: individual (1E 2259+586) — stars: pulsars - X-rays: bursts 


\section{Introduction}

Anomalous X-ray Pulsars (AXPs) and Soft Gamma Repeaters (SGRs) are two intriguing classes of isolated neutron stars, very likely magnetars, whose bright X-ray emission is powered by the decay of their strong magnetic fields. When the common nature of AXPs and SGRs was first proposed by Thompson \& Duncan (1996) with both being magnetars, the observational evidence linking them was tenuous. A major advance in connecting these two classes came when slow pulsations and rapid spin down, defining characteristics of AXPs, were discovered from SGR 1806-20 (Kouveliotou et al. 1998). Since then, further observational similarities among AXPs and SGRs have been established. For example, Marsden \& White (2001) performed a systematic analysis of SGR and AXP spectral data that showed the two classes form a continuum in spectral hardness versus spin-down rate where the SGRs have harder spectra and faster spin-down rates than the spectrally softer, slower braking AXPs. Similarly, the timing noise strength in SGRs and AXPs appears to be correlated with spin-down rate (Woods et al. 2000; Gavriil \& Kaspi 2002). For a review of SGRs and AXPs, see Kouveliotou (2003) and Mereghetti et al. (2002), respectively. Until recently, the most prominent characteristic distinguishing the two groups was the emission of soft, bright (up to $10^{44} \mathrm{ergs} \mathrm{s}^{-1}$ ) bursts of soft gamma-rays from the SGRs and not the AXPs. In fact, it was this extraordinary property of SGRs which led to doubt within the community that AXPs and SGRs were of the same nature. This uncertainty was removed when SGR-like bursts were recently discovered from two AXPs (Gavriil, Kaspi \& Woods 2002; Kaspi et al. 2003).

SGR-like bursts from the direction of an AXP were first detected from the source 1E 1048.1-5937 (Gavriil, Kaspi \& Woods 2002). A single weak SGR-like burst was detected during each of two Rossi X-ray Timing Explorer (RXTE) Proportional Counter Array (PCA) monitoring observations of this AXP separated by two weeks. However, the identification of $1 \mathrm{E}$ 1048.1-5937 as the burst source could not be made unambiguously due to the lack of imaging capability with the PCA. Interestingly, the quiescent properties of this AXP (e.g. energy spectrum, pulse profile, timing noise) most closely resemble those of the SGRs (Kaspi et al. 2001), making this AXP the most SGR-like of its class.

The second detection of SGR-like bursts was recorded from the AXP 1E 2259+586 on 2002 June 18. This is the least SGR-like of the AXPs in terms of its quiescent source properties. Unlike the two weak bursts observed earlier from 1E 1048.1-5937, this AXP showed a major SGR-like outburst, or collection of bursts (Kaspi et al. 2003). In total, more than 80 bursts were detected within 3 hours of observing time. A detailed study of these bursts will be presented in

a companion paper (Gavriil et al. 2003). In addition to emitting these hard X-ray bursts, several parameters of the persistent source changed in conjunction with this outburst, thereby confirming the AXP as the source of the burst emission (Kaspi et al. 2003).

Here, we present X-ray Multi-Mirror Mission (XMM-Newton) and RXTE PCA observations of the persistent X-ray flux from 1E 2259+586 before, during and after the 2002 June outburst. 
We quantify the changes of the spectral and temporal properties of the X-ray source as well as the time scales for their recovery. We compare the changes observed in this AXP to dynamic behavior seen in the persistent emission of SGR 1900+14 (Woods et al. 2001) and SGR 1627-41 (Kouveliotou et al. 2003) during episodes of intense burst activity. Finally, we present a possible explanation for the observed behavior in $1 \mathrm{E} 2259+586$ within the context of the magnetar model.

\section{XMM-Newton Observations}

The results presented in this section were obtained from observations of $1 \mathrm{E} 2259+586$ with the telescopes aboard the XMM-Newton observatory (Jansen et al. 2001). This observatory is comprised of three co-aligned X-ray telescopes. The focal plane instruments are one EPIC PN camera (Strüder et al. 2001) and two EPIC MOS cameras (Turner et al. 2001). All instruments are sensitive to X-rays between 0.2 and $15.0 \mathrm{keV}$. The PN camera has an effective area of $\sim 800 \mathrm{~cm}^{2}$ at $1.5 \mathrm{keV}$ while the MOS cameras each have areas of $\sim 350 \mathrm{~cm}^{2}$ at $1.5 \mathrm{keV}$. The focused X-ray beam for the telescopes serving each MOS camera is split between the focal plane instrument and the Reflection Grating Spectrometer (RGS [den Herder et al. 2001]).

The XMM-Newton observatory observed 1E 2259+586 five times during 2002. Three pointings were focused on different portions of CTB 109, the SNR surrounding 1E 2259+586. The primary scientific objective of the remaining two observations was the central point source. In each of the three CTB 109 pointings, the AXP is within the field-of-view at large off-axis angles $\left(10-13^{\prime}\right)$. Results from all five observations on the central source will be presented here. Analysis of the data from the remnant will be presented elsewhere (Sasaki et al., in preparation).

The first XMM-Newton observation of 1E 2259+586 was one of the remnant pointings, carried out on 2002 January 22. The second was centered on the AXP and was performed on 2002 June 11, fortuitously one week prior to the outburst of $1 \mathrm{E} 2259+586$. Following the outburst, a Target of Opportunity ( $\mathrm{ToO}$ ) was declared and the source was reobserved three days later on 2002 June 21. The remaining two observations of CTB 109 were carried out on 2002 July 09. See Table 1 for further details on these observations. Hereafter, each XMM-Newton observation will be referred to by the identifier label assigned to it in the first column of Table 1.

The two MOS cameras were operated in full frame mode for all but one observation (Obs3) in order to study the SNR. The frame time for this data mode is $2.6 \mathrm{~s}$ which causes severe pile-up for the $\operatorname{AXP}(z 50 \%)$. Therefore, these data are not considered further here.

The PN camera was operated in small window mode for both Obs2 and Obs3, the two observations closely bracketing the 2002 June outburst in time. The frame time for the PN camera in this mode is $\simeq 5.86 \mathrm{~ms}$, allowing detailed study of the pulsed emission. For the on-axis count rate of the AXP, the dead-time fraction is $30 \%$, but the pile-up fraction is only $0.03 \%$. The three off-axis pointings (Obs1, Obs4, and Obs5) were carried out in Extended Full-Frame mode which

has a time resolution of $200 \mathrm{~ms}$. The high off-axis angles for the AXP reduced the count rate by a 
Table 1: XMM-Newton observation log for 1E $2259+586$.

\begin{tabular}{|c|c|c|c|c|c|c|}
\hline Name & $\begin{array}{c}\text { XMM-Newton } \\
\text { Obsid }\end{array}$ & $\begin{array}{l}\text { Relation to } \\
\text { Burst Activity }\end{array}$ & $\begin{array}{c}\text { Date }^{a} \\
\text { (MJD TDB) }\end{array}$ & $\begin{array}{c}\text { PN Exposure } \\
(\mathrm{ksec})\end{array}$ & PN Data Mode ${ }^{c}$ & $\begin{array}{c}\text { AXP Offset }^{d} \\
\text { (arcmin) }\end{array}$ \\
\hline Obs1 & 0057540101 & Before & 52296.791 & 10.7 & Extended & 11.2 \\
\hline Obs2 & 0038140101 & Before & 52436.546 & 24.9 & Small & 1.7 \\
\hline Obs3 & 0155350301 & After & 52446.449 & 18.5 & Small & 1.7 \\
\hline Obs4 & 0057540201 & After & 52464.369 & 12.3 & Extended & 13.0 \\
\hline Obs5 & 0057540301 & After & 52464.606 & 12.4 & Extended & 9.7 \\
\hline
\end{tabular}

${ }^{a}$ Start time of each observation. Note that the outburst began on date 52443.65 MJD.

${ }^{b}$ Exposure times quoted reflect on-source times after filtering of flares, etc. for spectral and temporal analysis.

${ }^{c}$ Extended $=$ Extended Full Frame Mode; Small $=$ Small Window Mode.

$d$ The angular distance of $1 \mathrm{E} 2259+586$ from the optical axis of the EPIC mirrors.

factor $2-3$. The different data mode and reduction in count rate lessened the dead-time to $2 \%$, but the pile-up fraction increased to $3 \%$.

The RGS data from Obs2 and Obs3 were acquired in spectroscopy mode with $5.7 \mathrm{~s}$ time resolution and excellent energy resolution $(\Delta E / E=10-20)$. The coarse time resolution allowed for only phase-averaged spectral analysis (see $\S 2.2)$.

All data were processed using the XMM-Newton Science Analysis System ${ }^{8}$ (SAS) v5.4.1. For the PN data, the script epchain was run on the Observation Data Files. This script processes the data for use in higher-end analysis tools. A light curve of the full field-of-view (FOV) excluding the bright central source was constructed $(0.5-10.0 \mathrm{keV})$ and inspected for times of high background. We chose a threshold of 2 times the nominal background to define regions of high background. Periods of high background constituted $0-33 \%$ of each data set and were eliminated from further analysis.

\subsection{Pulse Timing Analysis}

We have used the data from the PN camera to study the pulsed emission from 1E 2259+586. Using the SAS tool evselect, events from within $10^{\prime \prime}$ and $12.5^{\prime \prime}$ radii circles around the position of the AXP were extracted for the on-axis and off-axis (remnant) pointings, respectively. Following the standard filtering procedures for $X M M-N e w t o n ~ P N$ data, grades $\geq 12$, and flag values equal 0

\footnotetext{
${ }^{8}$ http://xmm.vilspa.esa.es/external/xmm_sw_cal/sas.shtml
} 
were retained. Next, we filtered the event list on energy between 0.5 and $7.0 \mathrm{keV}$ to optimize the signal-to-noise ratio.

There is a known problem with the time tags of XMM-Newton PN data in which there can be sudden jumps in the photon arrival times of integer second values (W. Becker, private communication). It is still not understood at what stage of the processing these "time jumps" distort the time tags, only that they do occur. We searched for and identified four time jumps within two of the five data sets (Obs 2 and 3). All time jumps were identified in the two pointings where the PN camera was operated in small window mode. To find these time jumps we calculated the modulus of each event time stamp with the mean frame time of the corresponding data set. The frame time varied with data mode (small window vs. extended full frame) as well as between and within observations having identical data modes. The latter effect is believed to be due to temperature variations in the on-board electronics (XMM-Newton Helpdesk, private communication).

The time jumps in each data set were obvious after plotting the frame time residuals versus the time of each event. Time jumps manifested themselves as discontinuities in the frame time residuals plot. These discontinuities were identified and corrected for by adding or subtracting an integer number of seconds to the data following the jump until the frame time residuals matched precisely across the boundary. The time corrections we applied to the data were verified by the pulsar data. In the uncorrected pulsar data set, phase jumps were detected that were consistent with being equal to the time corrections required by the frame time inconsistencies. Using the time corrected data set, the phase jumps in the pulsar data disappeared. Therefore, we are secure about the relative timing of the corrected XMM-Newton PN data set. However, the absolute timing of these events will require a better understanding of the origin of the time jump problem.

The corrected time tags were next converted to the Solar system barycenter using the SAS tool barycorr. Assuming that the remaining uncertainty in the corrected time tags is a small integer number of seconds, the propagated error in the barycenter correction applied to the data is insignificant with respect to the precision with which we can measure the pulse frequency.

For the two point-source observations in small window mode (Obs2 and Obs3), the data were binned at twice the nominal frame time of each observation and a Fast Fourier Transform was constructed of the light curves $(0.5-7.0 \mathrm{keV})$. The only significant power detected between $4 \times$ $10^{-4} \mathrm{~Hz}$ and the Nyquist frequency $(44 \mathrm{~Hz})$ is from the first $7-8$ harmonics of the pulsar. The $3 \sigma$ upper limit to the mean fractional power in the frequency range $0.001-1.0 \mathrm{~Hz}$ (minus the pulsar frequency and harmonics) is $3 \times 10^{-3}$ and $2 \times 10^{-3}$ (RMS/MEAN) ${ }^{2} \mathrm{~Hz}^{-1}$, for Obs2 and Obs3 respectively. These limits are orders of magnitude lower than typical broadband noise power levels seen in accreting X-ray pulsars, even so-called "quiet" accreting pulsars such as 4U 1626-67 (Owens, Oosterbroek, \& Parmar 1997; Shinoda et al. 1990).

The precise pulse frequencies for each pointing were determined using a phase folding technique. For each data set, a template pulse profile was constructed by folding the data on 
the frequency as determined from the peak power of the fundamental frequency in the power density spectrum. Next, the data set was split into $\approx 10^{3} \mathrm{~s}$ segments and each segment was folded with the same frequency. The pulse profiles from each segment were cross-correlated with the template pulse profile and a phase offset was measured. The resulting phase differences for each segment were fit to a line and the slope was used to correct the frequency. Using the refined frequency, a new template pulse profile was constructed and the procedure for refining the pulse frequency was repeated. The epochs and frequencies measured for each XMM-Newton observation are listed in Table 2. Since two of the CTB 109 observations were performed sequentially, we determined a single frequency for the combined observation. The spin frequencies measured in the $X M M$-Newton observations are consistent with the much more precise spin ephemeris measured using the $R X T E$ data (see $\S 3.1$ ).

Table 2: Pulse frequencies and pulsed fractions of 1E 2259+586 measured using XMM-Newton PN data.

\begin{tabular}{|c|c|c|c|c|c|c|c|}
\hline \multirow[t]{2}{*}{ Observation } & \multirow{2}{*}{$\begin{array}{c}\text { Epoch } \\
\text { (MJD TDB) }\end{array}$} & \multirow{2}{*}{$\begin{array}{c}\text { Frequency }{ }^{a} \\
(\mathrm{~Hz})\end{array}$} & \multicolumn{5}{|c|}{ Pulsed Fraction } \\
\hline & & & $0.3-1.0 \mathrm{keV}$ & $1.0-2.0 \mathrm{keV}$ & $2.0-5.0 \mathrm{keV}$ & $5.0-12.0 \mathrm{keV}$ & $2.0-10.0 \mathrm{keV}$ \\
\hline Obs1 & $52296.791-52296.932$ & $0.14328688(55)$ & $0.169(15)$ & $0.195(6)$ & $0.230(10)$ & $0.314(73)$ & $0.232(10)$ \\
\hline Obs2 & $52436.546-52436.983$ & $0.14328705(13)$ & $0.215(6)$ & $0.225(3)$ & $0.234(4)$ & $0.284(23)$ & $0.234(4)$ \\
\hline Obs3 & $52446.449-52446.755$ & $0.14328746(9)$ & $0.168(5)$ & $0.200(2)$ & $0.223(3)$ & $0.189(13)$ & $0.220(3)$ \\
\hline $\mathrm{Obs} 4^{b}$ & $52464.369-52464.534$ & $0.14328771(13)$ & $0.166(15)$ & $0.180(7)$ & $0.225(11)$ & $0.294(50)$ & $0.230(10)$ \\
\hline Obs $5^{b}$ & $52464.606-52464.761$ & $0.14328771(13)$ & $0.139(13)$ & $0.168(6)$ & $0.225(8)$ & $0.339(38)$ & $0.230(8)$ \\
\hline
\end{tabular}

${ }^{a}$ Numbers in parenthesis indicate the $1 \sigma$ uncertainty in the least significant digits of the frequency.

${ }^{b}$ Frequency derived from combined data set of Obs4 and Obs5 due to their close proximity to one another in time.

Using the measured frequencies, the background-subtracted PN data were folded over different energy bands. The background was estimated by measuring the count rate for the different energy bands in a circular extraction region close to the pulsar and within CTB 109, but away from bright knots within the remnant. The normalized pulse profiles for different energy bands are shown in Figure 1. Clearly, the pulse profile changed significantly directly after the outburst. Subsequent X-ray observations revealed a gradual recovery to the pre-outburst pulse shape (see $§ 3.4$ ).

The root mean square (RMS) pulsed fractions were calculated for each observation over several energy bands. Following van der Klis (1989), the pulsed fractions were calculated using the first 7 harmonics of the Fourier representation of the pulse profile according to the equation

$$
F_{R M S}=\sqrt{\sum_{k=1}^{7} \frac{\alpha_{k}^{2}+\beta_{k}^{2}-\left(\sigma_{\alpha_{k}}^{2}+\sigma_{\beta_{k}}^{2}\right)}{2}}
$$

where 


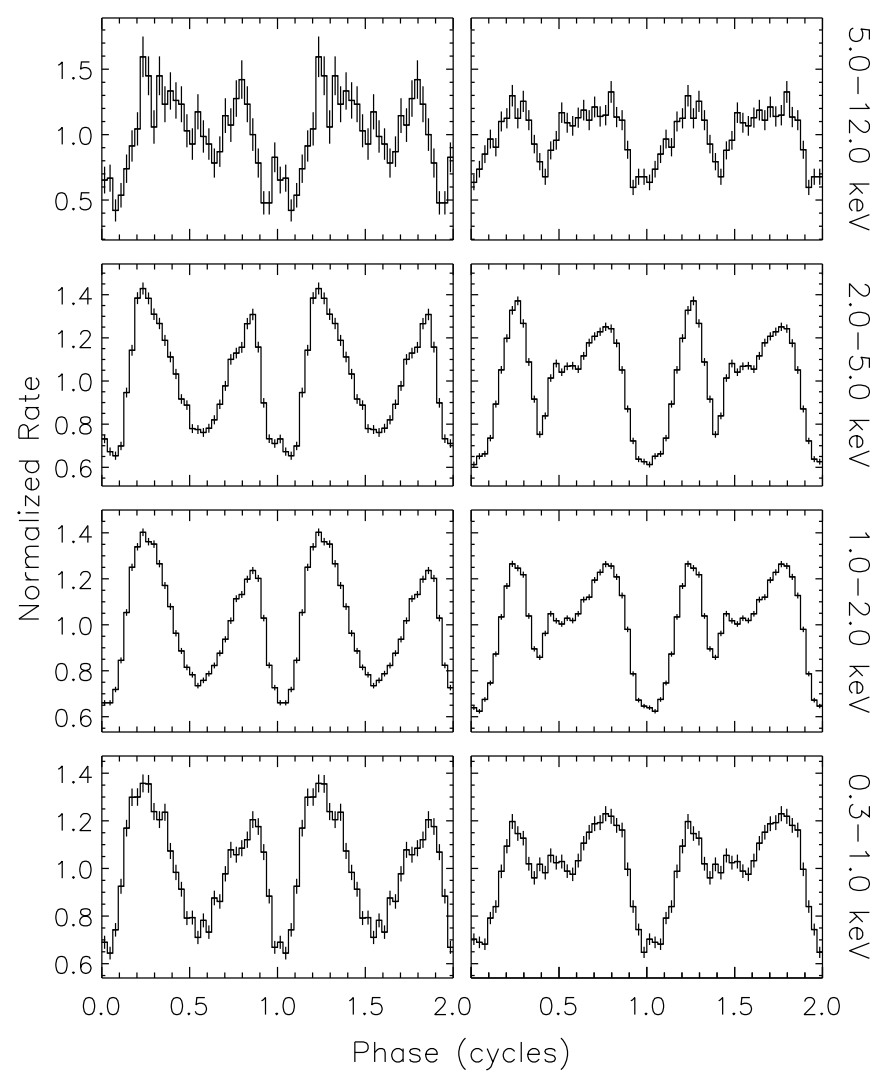

Fig. 1. - The folded pulse profile of 1E $2259+586$ as observed with the XMM-Newton PN camera as a function of energy for the two observations nearest the 2002 June outburst. The series of panels on the left are the folded pulse profiles directly pre-outburst (Obs2). The right column of panels contain the pulse profiles from the observation directly following the burst activity (Obs3). See Table 1 for exact times of observations.

$$
\begin{gathered}
\alpha_{k}=\frac{1}{N} \sum_{i=1}^{N} r_{i} \cos 2 \pi \phi_{i} k, \quad \beta_{k}=\frac{1}{N} \sum_{i=1}^{N} r_{i} \sin 2 \pi \phi_{i} k, \\
\sigma_{\alpha_{k}}^{2}=\frac{1}{N} \sum_{i=1}^{N} \sigma_{r_{i}}^{2} \cos ^{2} 2 \pi \phi_{i} k, \quad \sigma_{\beta_{k}}^{2}=\frac{1}{N} \sum_{i=1}^{N} \sigma_{r_{i}}^{2} \sin ^{2} 2 \pi \phi_{i} k .
\end{gathered}
$$

Here, $F_{R M S}$ is the pulsed fraction, $k$ refers to the harmonic number, $i$ refers to the phase bin, $N$ is the total number of phase bins, $\phi_{i}$ is the phase, $r_{i}$ is the count rate in the $i^{\text {th }}$ phase bin, and $\sigma_{x_{i}}$ 
is the uncertainty in the count rate of the $i^{\text {th }}$ phase bin. The values and uncertainties are given in Table 2.

Directly following the outburst (Obs3), the pulsed fraction at all energies dropped relative to the pre-outburst level (Obs2). The largest change was seen in the hard band (5.0-12.0 keV). During the $X M M$-Newton observations three weeks after the outburst, the broadband pulsed fraction $(2-10 \mathrm{keV})$ had recovered to its pre-outburst value, although the pulse profile was still significantly different. The time evolution of the pulsed fraction is presented in $\S 3.3$ where additional pulsed fraction measurements made with the RXTE PCA are reported.

In the observation directly before the outburst (Obs2), the pulsed fraction does not vary strongly with photon energy. The energy dependence of the pulsed fraction is most prominent in the observations following the outburst where the pulsed fraction increased significantly with energy. Interestingly, the pulsed fraction in the soft band $(0.3-1.0 \mathrm{keV})$ is higher one week before the outburst than at any of the other XMM-Newton epochs before or after.

\subsection{Spectroscopy}

The PN data were used for spectral analysis of 1E 2259+586 because of their excellent signal-to-noise ratio and negligible pile-up. For the two observations centered on the point source, events from within a $10^{\prime \prime}$ radius circle around the position of the AXP were extracted using the SAS tool evselect. Following the standard procedures for XMM-Newton PN data, these events were filtered for grades $\geq 4$, and flag values equal 0 were retained for the source spectrum. Similarly, a background spectrum was extracted using the same filtering criteria from nearby circular regions of $10^{\prime \prime}$ radius for each observation identical to the regions used for the pulsed fraction analysis. The total number of source counts accumulated for each spectrum was 259,000 and 373,000 for the pre-burst (Obs2) and post-burst (Obs3) observations, respectively.

The three observations of CTB 109 included the AXP, but at large off-axis angles $\left(10-13^{\prime}\right)$. At off-axis positions of $\sim 10^{\prime}$, the point spread function of the EPIC mirrors is significantly broader than on axis. Due to this effect, a larger source extraction radius (12.5") was used. Background spectra were extracted from circular regions of radius $\sim 22^{\prime \prime}$ with centers at off-axis positions similar to the source extraction region. The larger extraction radii were chosen to increase the number of background counts and improve the accuracy of the background subtraction. Furthermore, the background spectra were extracted from the same chip. Due to the off-axis positions of the AXP

and the lower exposures of the CTB 109 observations, the total number of source counts extracted in each spectrum was much lower, between 38,000 and 56,000.

Each spectrum was grouped such that there was a minimum of 25 counts per bin. The spectra 
were fit individually using $\mathbf{X S P E C}^{9}$ v11.2.0. Due to the high column along the line of sight to this source, the observed counts in Pulse Height Amplitude (PHA) channels corresponding to $E<0.6$ $\mathrm{keV}$ are dominated by the low PHA tails of events whose true energies are above $1 \mathrm{keV}$. We, therefore, restrict our spectral fits to the data in the range $0.6-12.0 \mathrm{keV}$. Fits to single component models (blackbody, power law and bremsstrahlung) modified by interstellar absorption did not yield statistically acceptable fits. The resulting reduced $\chi^{2}$ values to the single component models were $z 4$. We next tried the standard AXP two-component spectral model of a blackbody plus a power law modified by interstellar absorption. We obtained good fits to all five data sets. The results of these fits are given in Table 3. The X-ray spectrum of $1 \mathrm{E} 2259+586$ during Obs2 and the residuals from the best fit are shown in Figure 2. Formally, the fit to the Obs3 spectrum is not statistically acceptable. However, the residuals between 0.8 and $3.0 \mathrm{keV}$ constitute the majority of the total $\chi^{2}$ and are at the few percent level. These residuals are very likely due to uncertainties in the instrumental response (Haberl et al. 2003). No narrow line features are evident in any of the phase-averaged spectra. Within the energy range $0.9-2.0 \mathrm{keV}$, the $90 \%$ confidence limit on a narrow line feature of the order of the PN response function $(\sim 50 \mathrm{eV})$ is $\sim 10 \mathrm{eV}$ for the equivalent width. Between 2.0 and $7.0 \mathrm{keV}$, the response function increases up to $\sim 130 \mathrm{eV}$ and the line limit increases from $\sim 10 \mathrm{eV}$ to $\sim 70 \mathrm{eV}$.

Table 3: Phase-averaged spectral fit parameters of 1E 2259+586 from XMM-Newton PN data.

\begin{tabular}{cccccc}
\hline \hline Parameter & Obs1 & Obs2 & Obs3 & Obs4 & Obs5 \\
\hline$N_{H}\left(10^{22} \mathrm{~cm}^{-2}\right)$ & $1.096(24)$ & $1.098(12)$ & $1.035(8)$ & $0.953(24)$ & $0.937(20)$ \\
$k T(\mathrm{keV})$ & $0.488(14)$ & $0.411(4)$ & $0.517(5)$ & $0.537(19)$ & $0.548(13)$ \\
$\Gamma$ & $4.04(8)$ & $4.10(3)$ & $3.59(2)$ & $3.62(7)$ & $3.58(6)$ \\
Flux $^{b}\left(10^{-11}\right.$ ergs cm $\left.^{-2} \mathrm{~s}^{-1}\right)$ & $1.24(4)$ & $1.30(2)$ & $3.47(3)$ & $2.01(5)$ & $2.11(5)$ \\
Unabs Flux $^{c}\left(10^{-11} \mathrm{ergs} \mathrm{cm}^{-2} \mathrm{~s}^{-1}\right)$ & $1.53(4)$ & $1.63(2)$ & $4.17(4)$ & $2.37(6)$ & $2.49(5)$ \\
$\mathrm{PL} / \mathrm{BB} \mathrm{ratio}^{d}$ & $0.70(6)$ & $0.43(2)$ & $0.74(2)$ & $0.93(9)$ & $0.79(6)$ \\
$\chi^{2} /$ dof & $619 / 578$ & $922 / 851$ & $1332 / 1094$ & $606 / 540$ & $599 / 638$ \\
\hline \hline
\end{tabular}

${ }^{a}$ Numbers in parentheses indicate the $1 \sigma$ uncertainty in the least significant digits of the spectral parameter.

${ }^{b}$ Observed flux from both spectral components $2-10 \mathrm{keV}$.

${ }^{c}$ Unabsorbed flux from both spectral components $2-10 \mathrm{keV}$.

$d$ The ratio of the power-law flux $(2-10 \mathrm{keV})$ to the bolometric blackbody flux (corrected for absorption).

We next included the RGS data in our spectral analysis. The RGS spectra for Obs2 and Obs3 were extracted using standard processing techniques for a point source. The data were grouped to 25 counts per spectral bin and ported into XSPEC for simultaneous fitting with the PN data.

\footnotetext{
${ }^{9}$ http://heasarc.gsfc.nasa.gov/docs/xanadu/xspec/
} 


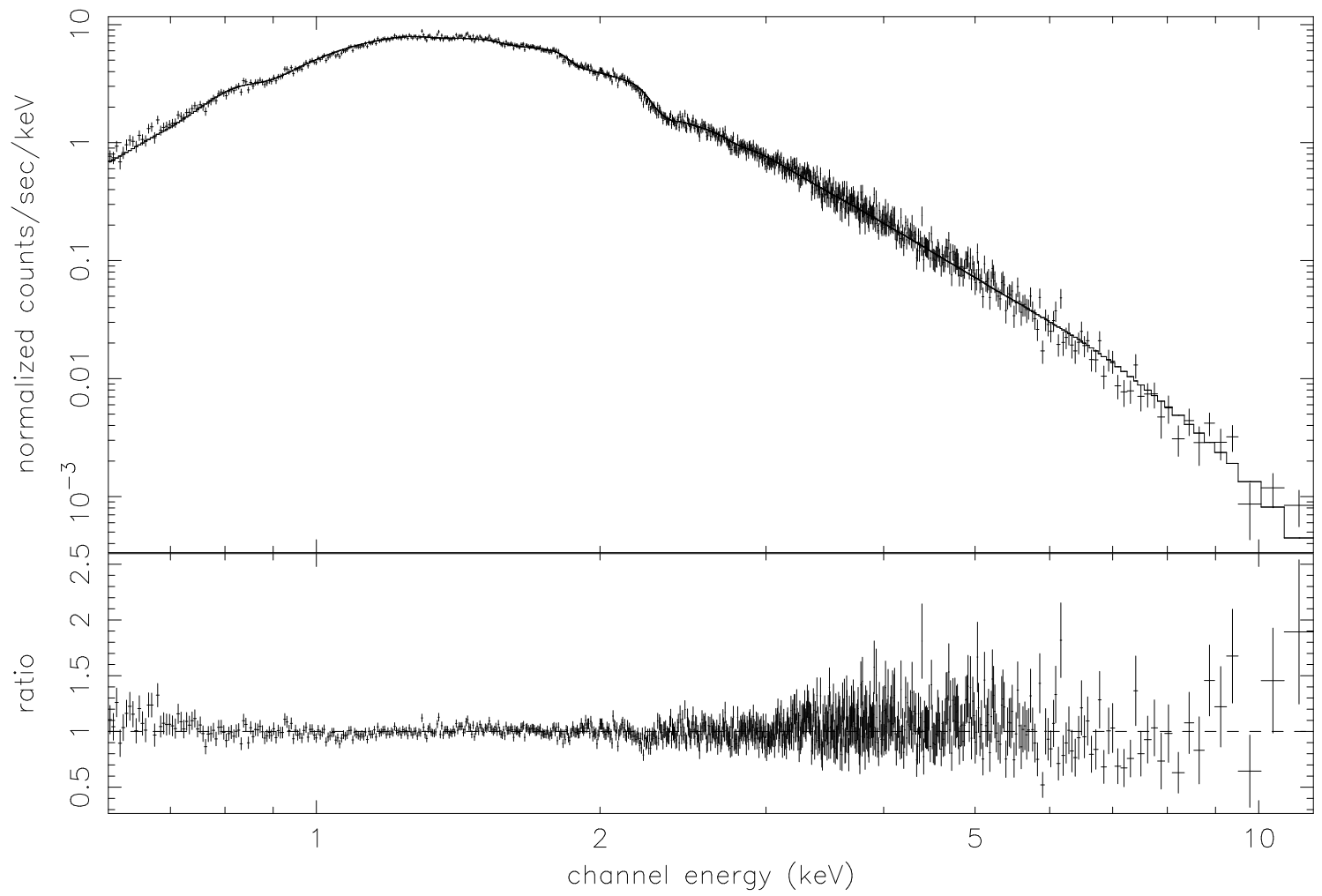

Fig. 2. - The phase-averaged X-ray spectrum of $1 \mathrm{E} 2259+586$ as observed with the XMM-Newton PN camera during the observation one week prior to the 2002 June outburst (Obs2). The ratio of the data to the best-fit model (see Table 3) folded through the instrumental response is shown in the bottom panel.

Each observation was fit independently, again to the blackbody plus power law model, and the measured spectral parameters were consistent with those obtained using the PN data alone. The superior energy resolution of the RGS data combined with the high signal-to-noise data from the PN camera allow us to put even more constraining limits on the presence of narrow line features. Within the energy range $0.8-1.75 \mathrm{keV}$, the $90 \%$ confidence limit on a narrow line feature $(\sim 3-10$ $\mathrm{eV})$ is $\sim 7 \mathrm{eV}$ for the equivalent width.

We used the RGS data to confirm whether the fit value of $N_{H}$ was affected by the steep power-law component of the spectral model. Specifically, using just the PN data, it is difficult to distinguish between a steep power law that is strongly absorbed and a flat or inverted power law (e.g. blackbody) that is observed through modest absorption. The Ne-K edge at $0.87 \mathrm{keV}$ is readily detected in the RGS data. Freeing both the $N_{H}$ and the Ne abundance relative to solar, $a_{N e}$, using the tbvarabs model in XSPEC, we find that $a_{N e}=1.27 \pm 0.24$ (at $90 \%$ confidence for one degree of freedom) and the best-fit value of $N_{H}$ is very close to the best-fit value when $a_{N e}$ is fixed at 1 . Thus, unless the true Ne abundance is significantly different from the solar value, the 
fitted $N_{H}$ seems accurate (see Table 3 ).

Clearly, the energy spectrum of 1E 2259+586 hardened following the 2002 June outburst. Between the XMM-Newton observation one week prior to the outburst and three days following, the photon index became significantly flatter and the blackbody temperature rose. However, the blackbody temperature during Obs1, several months before the outburst, was significantly higher than the Obs2 temperature and only marginally lower than the post-outburst temperature, albeit with a considerably lower flux. This might suggest that the low temperature measured during Obs2 was an indicator of the impending outburst. However, the blackbody temperature measured using the Chandra X-ray Observatory in 2000 January was $0.412 \pm 0.006 \mathrm{keV}$ (Patel et al. 2002), and there was no glitch detected from $1 \mathrm{E} 2259+586$ at this time (Gavriil \& Kaspi 2002). Moreover, Patel et al. (2002) measure a photon index of $3.6 \pm 0.1$, consistent with the post-outburst value. Other previous observations of this AXP have shown similar variance in these spectral parameters (e.g. Parmar et al. 1998; Marsden \& White 2001), in addition to the PL/BB flux ratio (Marsden \& White 2001). Thus, it appears that 1E 2259+586 undergoes significant spectral changes independent of large outbursts and the spectral parameters measured during all of the XMM-Newton observations (even those following the outburst) are within the historical range of these parameters.

Using the frequencies given in the last section, we extracted spectra for 16 phase intervals per cycle per observation. For the observation one week prior to the outburst (Obs2), between 10,000 and 22,000 counts were accumulated per phase interval. The spectrum having the least number of total counts (i.e. pulse minimum) was grouped such that at least 25 counts were contained in each data bin. The grouping for each of the remaining data sets was forced to be identical to the grouping used for pulse minimum. As with the phase-averaged spectrum, we fit a blackbody plus a power law modified by interstellar absorption. The sixteen intervals were fit simultaneously with the column density forced to be the same for all intervals. The measured column density is consistent with the phase-averaged value. All other parameters were allowed to vary in the fit. The folded pulse profile, photon index, blackbody temperature, and ratio of power-law to blackbody flux are shown in Figure 3. The average values of the measured spectral parameters are denoted by the horizontal dashed line shown within each panel. The flux ratio uncertainties are determined by propagating the flux errors in each spectral component. The probability that the variance in the spectral parameters with phase is purely statistical is $1.4 \times 10^{-15}, 0.10$, and $<10^{-16}$ for the photon index, blackbody temperature, and the flux ratio of the two components. The superb statistics of the PN data enabled us to identify both the photon index and flux ratio as varying significantly as a function of pulse phase whereas the blackbody temperature is consistent with remaining constant.

Taken at face value, this observation favors two distinct components to the $1 \mathrm{E} 2259+586$ spectrum as has been argued previously (Thompson \& Duncan 1996; Perna et al. 2001). However, the two components are likely highly correlated in order to explain the minimal variation of the pulse fraction and shape versus energy (Özel, Psaltis, \& Kaspi 2001). Alternatively, a single 
thermal component modified by the strong magnetic field (Özel 2001) may be possible if there is substantial variation in the magnetic field across the stellar surface.
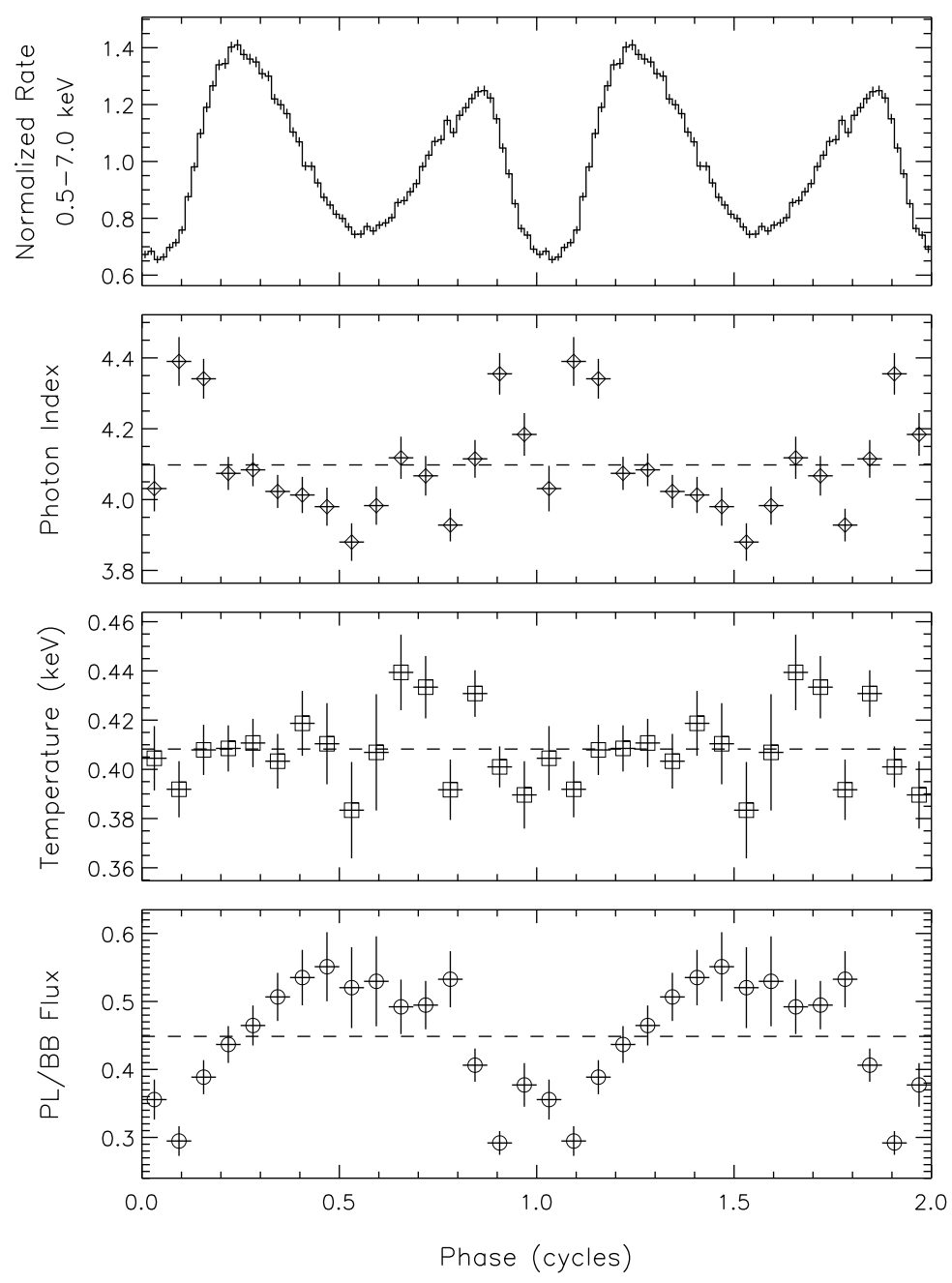

Fig. 3. - The phase resolved X-ray spectrum of $1 \mathrm{E} 2259+586$ as observed with the XMM-Newton PN camera seven days prior to the observed burst activity (Obs2). From the top panel down: the folded pulse profile over the energy range $0.5-7.0 \mathrm{keV}$, the photon index, the blackbody temperature $(k T)$, the ratio of power-law to blackbody flux. The horizontal dashed lines in the bottom three panels denote the average value for each spectral parameter. For the ratio of power-law to blackbody flux, the power-law flux was summed over the energy range $2-10 \mathrm{keV}$ and the blackbody was summed over all photon energies, each corrected for the interstellar absorption (see text for value). 
The same analysis procedure was applied to the data directly following the outburst. The folded profile and spectral parameters versus pulse phase are shown in Figure 4. Unlike the observation 10 days earlier, the changes in photon index are only marginally significant $(2.9$ $\left.\times 10^{-3}\right)$. Similarly, the blackbody temperature changes are also marginally significant $(1 \times$ $\left.10^{-3}\right)$. The flux ratio, on the other hand, still shows significant variability $\left(<10^{-16}\right)$. The phase dependence of the flux ratio data is markedly different than what was seen pre-outburst.

The total number of counts recorded from 1E $2259+586$ within the off-axis pointings (Obs1/4/5) is comparable to the counts recorded within only two phase bins for the on-axis pointings. Due to the poorer count statistics in these observations, phase resolved spectroscopy was not performed on these data.

\section{RXTE PCA Observations}

The bursting behavior on 2002 June 18 from 1E 2259+586 (Figure 5) was detected as part of an ongoing monitoring campaign of AXPs spanning the last six years (e.g. Kaspi, Chakrabarty \& Steinberger 1999; Gavriil \& Kaspi 2002). Follow-up ToO observations of the source with the PCA were immediately triggered and started as early as one day following the outburst. ToO observations continued for roughly the next month before regular monitoring observations resumed. Here, we report on observations of the persistent and pulsed emission from the AXP leading up to, during and following the 2002 outburst of this source. A detailed analysis of the burst properties is presented in a companion paper (Gavriil et al. 2003).

The PCA instrument aboard RXTE is a collimated (1 deg FWHM FOV) proportional counter containing a mixture of xenon and methane gas (Jahoda et al. 1996). It is sensitive to X-rays in the 2-60 keV bandpass and has a maximum effective area of $\sim 6500 \mathrm{~cm}^{2}$ at $7 \mathrm{keV}$. The data from the PCA are read out in a number of different data acquisition modes. Different data modes were used depending upon the analysis performed. In all of the analysis, integration times including detected burst emission were eliminated from the accumulated spectra and/or light curves.

With the exception of the first two ToO pointings directly following the outburst, GoodXenonWithPropane data were acquired during each observation. These data were used for the pulse timing analysis described below. For the first two ToO observations, data modes better

suited for studying bright bursts were employed, however, no further burst activity was seen. For these pointings, the event data mode (E_125us_64M_0_1s) was used for the timing analysis.

Starting from RXTE production-level data, GoodXenonWithPropane and event mode data were energy selected $(2-10 \mathrm{keV})$ for all Xenon layers and binned into light curves having 0.0625 $\mathrm{s}$ time resolution. The time values in the light curve were then corrected to the solar system barycenter. For these processing steps, the standard prescriptions for RXTE PCA data analysis 

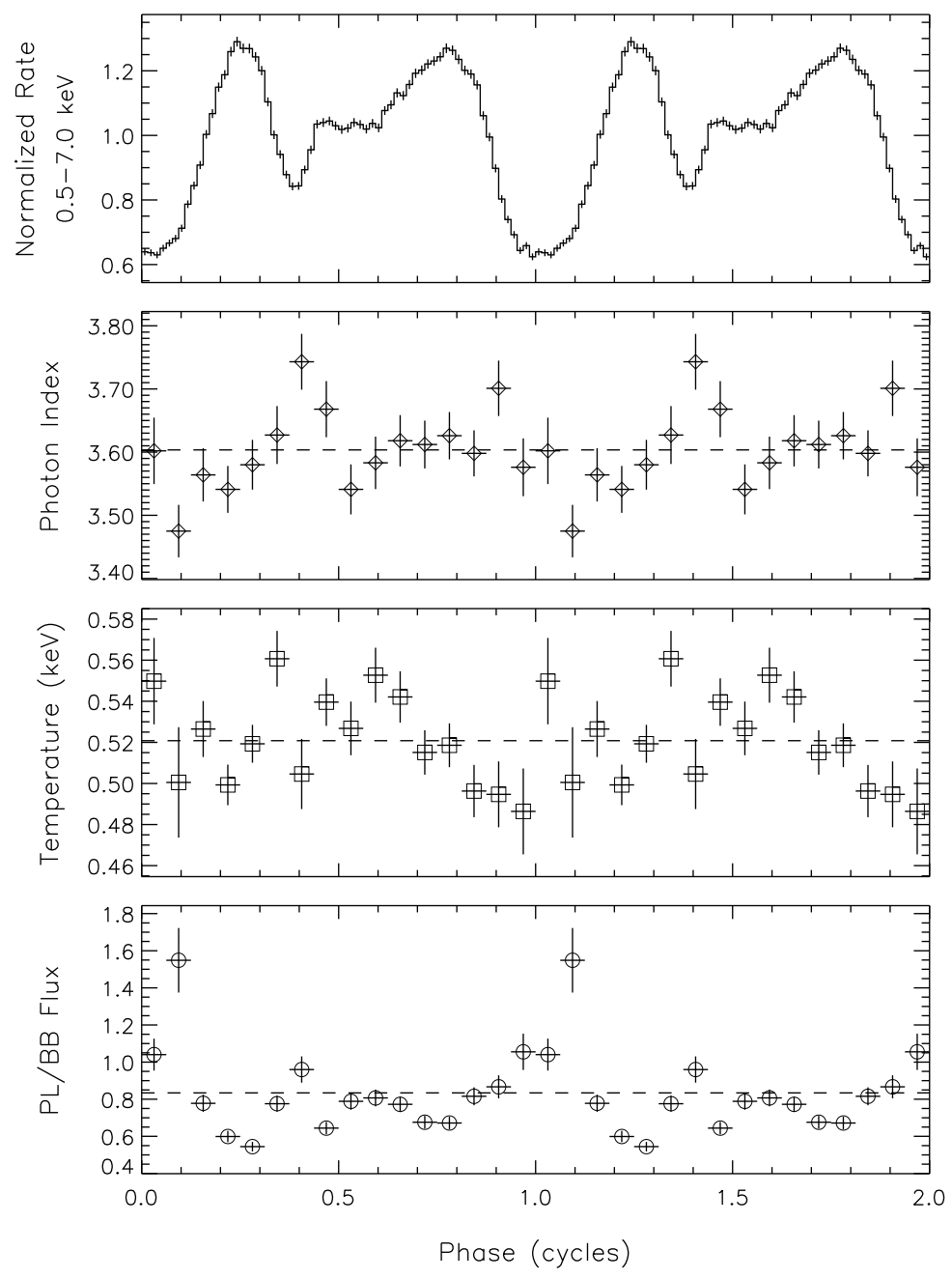

Fig. 4. - The phase resolved X-ray spectrum of $1 \mathrm{E} 2259+586$ as observed with the XMM-Newton PN camera three days after the observed burst activity (Obs3). From the top panel down: the folded pulse profile over the energy range $0.5-7.0 \mathrm{keV}$, the photon index, the blackbody temperature $(k T)$, the ratio of power-law to blackbody flux. The horizontal dashed lines in the bottom three panels denote the average value for each spectral parameter. For the ratio of power-law to blackbody flux, the power-law flux was summed over the energy range $2-10 \mathrm{keV}$ and the blackbody was summed over all photon energies, each corrected for the interstellar absorption (see text for value).

were followed ${ }^{10}$. The data were filtered further by eliminating times of high background and bursts

${ }^{10}$ http://heasarc.gsfc.nasa.gov/docs/xte/recipes/cook_book.html 


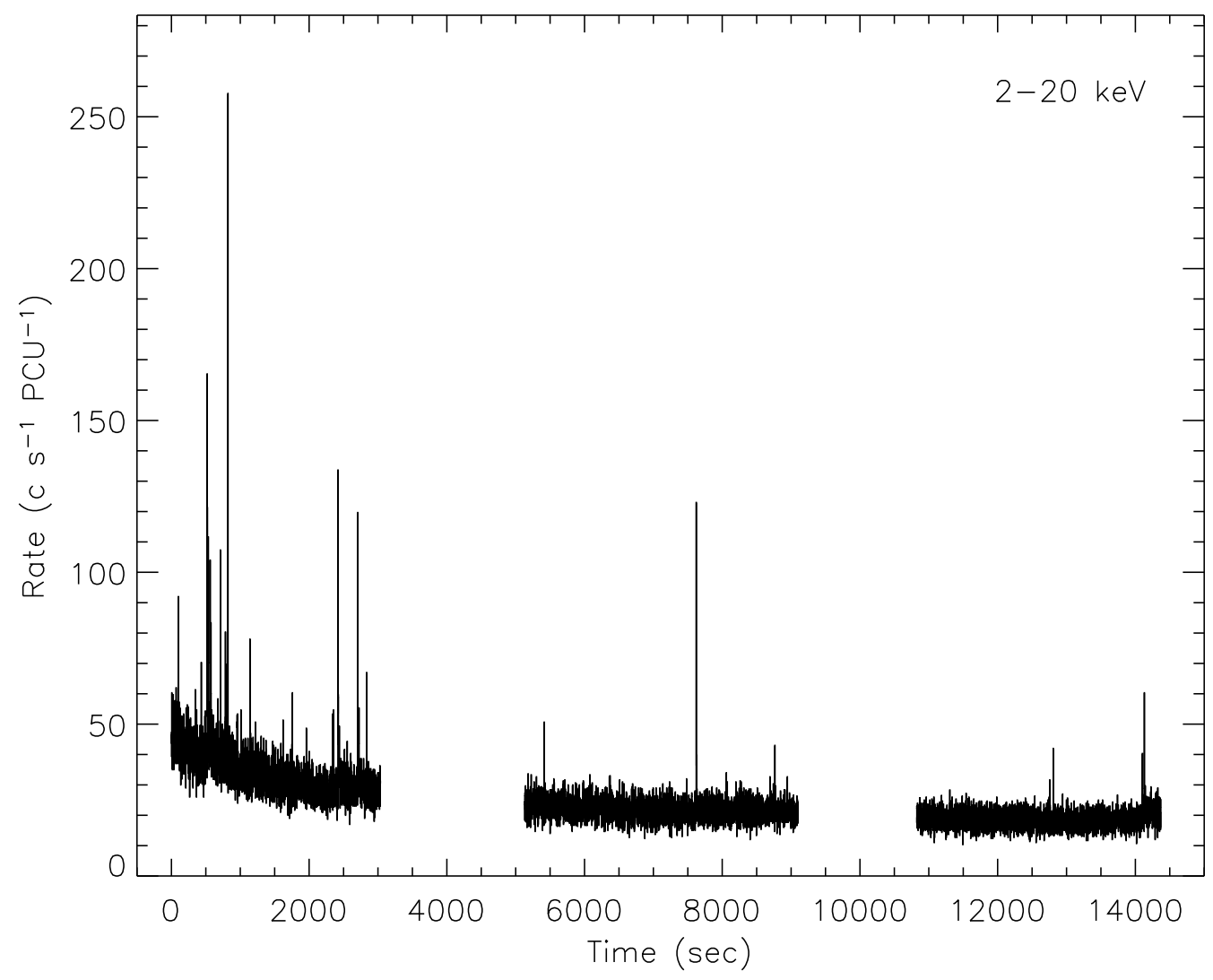

Fig. 5.- RXTE PCA light curve of 1E 2259+586 recorded on 2002 June 18. The light curve displays counts within $2-20 \mathrm{keV}$ at 1 -s time resolution.

during the 2002 June 18 observation using custom software. The count rates were normalized to the number of PCUs on at a given time.

\subsection{Pulse Timing Analysis}

Within two days of the outburst, it was clear from the PCA data that a glitch had occurred (Kaspi et al. 2003). A new timing ephemeris was established and refined with continuing PCA observations. It gradually became apparent that accompanying the sudden increase in frequency was a dramatic increase in spin-down rate (i.e. torque) by a factor $\sim 2$. This torque excess decayed rapidly over the next several weeks, approaching the pre-outburst level. Here, we extend the pulse timing ephemeris 15 months beyond the glitch, allowing us to better characterize the frequency evolution post-glitch and quantify the recovery time scale of the torque. 
As with the $X M M$-Newton PN data, a phase folding technique was used to determine the precise ephemeris from the $R X T E$ PCA data. Briefly, photon arrival times, obtained using data in the energy range $2-10 \mathrm{keV}$ were binned with $62.5-\mathrm{ms}$ time resolution, reduced to the solar system barycenter, then folded at the nominal pulse period. The folded pulse profiles for each pointing were cross-correlated in the Fourier domain with a template pulse profile obtained from pre-outburst observations only and a relative phase (i.e. TOA) was measured. Our analysis includes a total of 62 phase measurements obtained between 2000 March and 2003 September, with 43 measurements obtained either during or post-outburst.

This particular analysis was complicated by the change in the pulse profile at the time of the outburst (see §3.4), as the cross-correlation procedure assumes a fixed profile. Gross pulse profile changes in which the relative amplitudes of the two peaks were reversed resulted in a misidentification of the standard fiducial point by the cross-correlator for a subset of the phase measurements made during the outburst. This was accounted for by aligning the average pulse profile during the outburst observation with the template profile. The aligned outburst profile was then used as the template for phase measurements during the outburst. We verified this procedure by comparing the phase measurements made using this method to those calculated using the original template. We found that where the cross-correlator identified the appropriate peak in the original measurements, the revised measurements agreed to within the errors. We further checked that our method worked by verifying that the results we describe below are insensitive to the omission of the phase measurements made within 1 day of the burst activity where the pulse profile changes were largest.

We attempted to fit the spin evolution through and following the glitch with standard glitch models, that is, a simple jump in frequency, and a two-component frequency jump in which one part decays exponentially (Eq. 2 below with $\Delta \nu_{g}=0$ ). Neither provided a good fit to the phase data. Figure 6 shows the frequency evolution and phase residuals following subtraction of the best-fit model including a sudden frequency jump and an additional exponential decay. The best-fit parameters imply a total frequency jump $(\Delta \nu)$ of $6.3 \times 10^{-7} \mathrm{~Hz}$, with a fraction $Q=0.23$ decaying on a time scale of $\sim 40$ days. However, as can be seen from the Figure, the residuals from this model show a significant systematic trend at the few percent level $\left(\chi^{2}=447\right.$ for 54 dof $)$. Omitting the immediate post-burst data in which the pulse profile had substantially changed does not alter the result. It is possible, however, that the residuals are a result of low-level systematic pulse morphology variations (see $\S 3.4$ ). This is hard to rule out.

However, as we show next, a model in which a substantial portion of the glitch is resolved in time provides a better fit to the data. We employed a more complex model previously invoked for fitting two glitches from the Crab pulsar in which the rise in frequency is resolved (Lyne, Pritchard, \& Graham-Smith 1993; Wong, Backer \& Lyne 2001). We model the frequency evolution as 


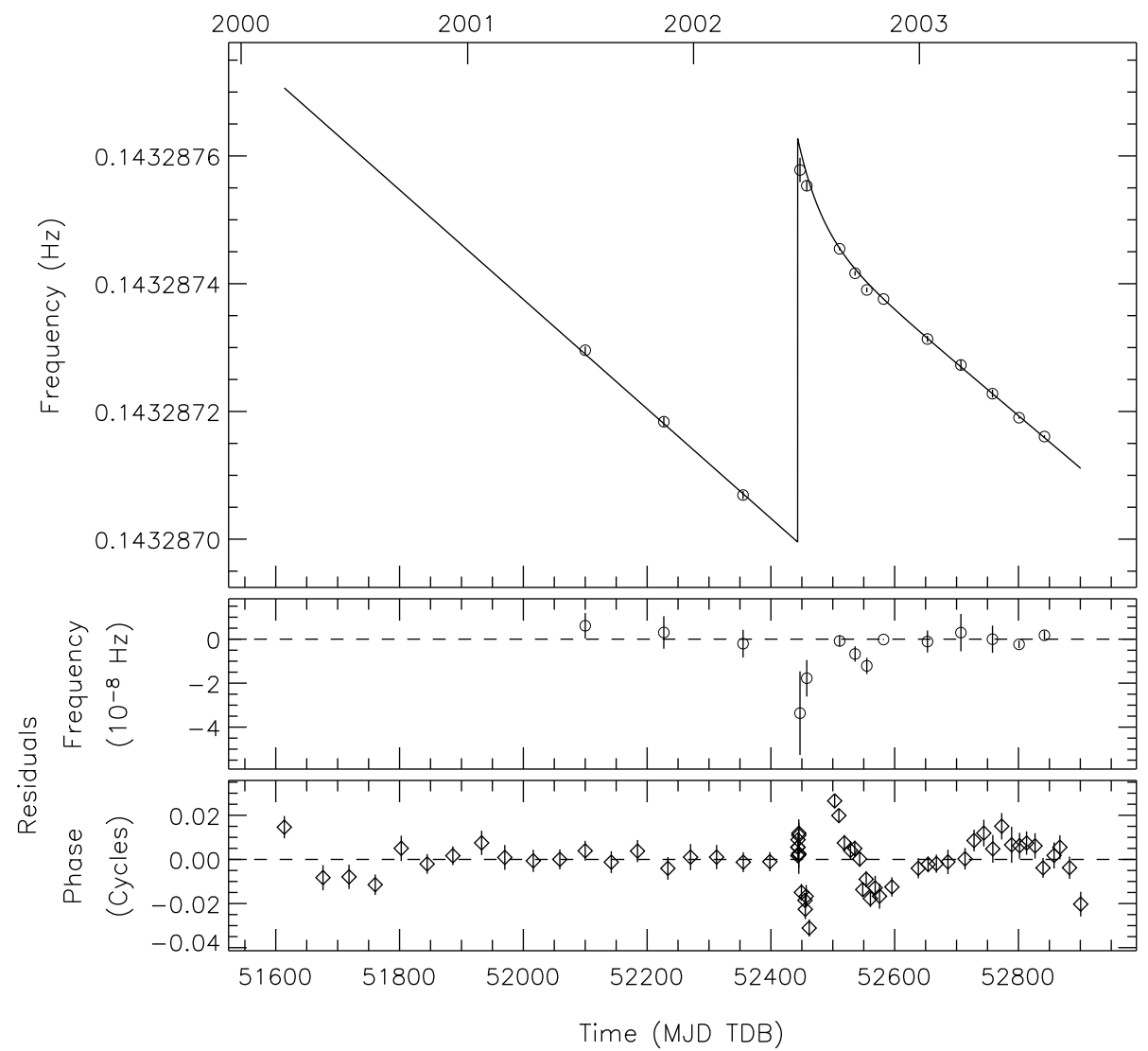

Fig. 6. - Top Panel - Frequency evolution of 1E 2259+586 around the time of the outburst for a simple exponential recovery model. See text for details. The solid line represents the best-fit model. The circles denote frequency measurements for independent subsets of data. Middle Panel - The frequency residuals of the independent frequency points minus the model. Bottom Panel-Phase residuals with respect to the best-fit model. Clearly, there is a systematic trend in the post-glitch residuals reflecting the inadequacy of this model.

$$
\nu=\nu_{0}(t)+\Delta \nu+\Delta \nu_{g}\left(1-e^{-\left(t-t_{g}\right) / \tau_{g}}\right)-\Delta \nu_{d}\left(1-e^{-\left(t-t_{g}\right) / \tau_{d}}\right)+\Delta \dot{\nu} t
$$

where $\nu_{0}(t)$ is the frequency evolution pre-glitch, $\Delta \nu$ is a instantaneous frequency jump, $\Delta \nu_{g}$ is the resolved frequency jump that grows exponentially on a time scale $\tau_{g}, \Delta \nu_{d}$ is the post-glitch frequency drop that decays exponentially on a time scale $\tau_{d}, t_{g}$ is the glitch epoch, and $\Delta \dot{\nu}$ is the post-glitch change in the long-term frequency derivative.

The full ensemble of phases was fit to the model above using a Levenberg-Marquardt least 
squares fitting routine. The fit improved significantly over the simple jump in frequency and partial exponential recovery $\left(\chi^{2}=131\right.$ for 53 dof $)$. Due to the strong correlation between the amplitudes of the two exponential factors $\left(\Delta \nu_{g}\right.$ and $\left.\Delta \nu_{d}\right)$ and similar associated time scales, our fit was mildly non-convergent. For this reason, we can only quote lower limits for each amplitude. Fixing either one of the exponential amplitudes to a value above its lower limit allows the fit to converge. The key parameter is the difference between the two amplitudes which determines the peak frequency following the glitch. The best-fit parameters for this fit (including the relationship between $\Delta \nu_{g}$ and $\left.\Delta \nu_{d}\right)$ are given in Table 4 . The variance of the time scales $\left(\tau_{g}\right.$ and $\left.\tau_{d}\right)$ is far less than the amplitudes. We quote the formal errors for these time scales using a fixed $\Delta \nu_{g}=$ $2.3 \times 10^{-6} \mathrm{~Hz}$. However, fixing $\Delta \nu_{g}$ to the minimum allowed value yields significantly different time scales $\left(\tau_{g}=12.8(7)\right.$ days and $\tau_{d}=17.4(6)$ days). Going to higher values for $\Delta \nu_{g}$ (and correspondingly $\Delta \nu_{d}$ ) does not change the time scales significantly.

Table 4: Spin Parameters for 1E 2259+586 from 3.2 years of Phase-Coherent Timing using $R X T E$ PCA data.

\begin{tabular}{lc}
\hline \hline Spin Frequency $^{a}, \nu(\mathrm{Hz})$ & $0.14328703257(21)$ \\
Spin Frequency Derivative, $\dot{\nu}\left(\mathrm{Hz} \mathrm{s}^{-1}\right)$ & $-9.920(6) \times 10^{-15}$ \\
Epoch (MJD TDB) & 52400.0000 \\
\hline$\Delta \nu(\mathrm{Hz})$ & $5.25(12) \times 10^{-7}$ \\
$\Delta \nu_{g}^{b}(\mathrm{~Hz})$ & $>8.7 \times 10^{-7}$ \\
$\tau_{g}($ days $)$ & $14.1(7)$ \\
$\Delta \nu_{d}(\mathrm{~Hz})$ & $\Delta \nu_{g}+\sim 5 \times 10^{-9}$ \\
$\tau_{d}($ days $)$ & $15.9(6)$ \\
$\Delta \dot{\nu}\left(\mathrm{Hz} \mathrm{s}{ }^{-1}\right)$ & $+2.18(25) \times 10^{-16}$ \\
$t_{g}(\mathrm{MJD}$ TDB) & $52443.13(9)$ \\
\hline RMS Timing Residual (ms) & 44.9 \\
Start Observing Epoch (MJD) & 51613 \\
End Observing Epoch (MJD) & 52900 \\
\hline \hline
\end{tabular}

${ }^{a}$ Numbers in parentheses represent $1 \sigma$ uncertainties in the least significant digits quoted.

${ }^{b}$ Lower limit given at $90 \%$ confidence.

The frequency evolution of $1 \mathrm{E} 2259+586$ just before and following the outburst as determined by our fit is shown in Figure 7. The pre-outburst ephemeris is fully consistent with the ephemeris determined through earlier monitoring of this pulsar (Gavriil \& Kaspi 2002). The glitch epoch $\left(t_{g}\right)$ precedes the PCA observation containing the burst activity by $12.5 \pm 2.1$ hours. Note that the glitch epoch precedes the observed burst activity whether the exponential growth term is included in the fit or not (it is $4.7 \sigma$ in the case with no growth term). The exponential growth term clearly improves the fit to the full data set; however, there is a systematic trend in the 
phase residual cluster just post-glitch. This is discussed in detail below. Including the growth term significantly reduces the time scale of the exponential decay term to $\sim 16$ days. Finally, the long-term post-glitch spin-down rate decreases significantly in magnitude $(8 \sigma)$ for both models.

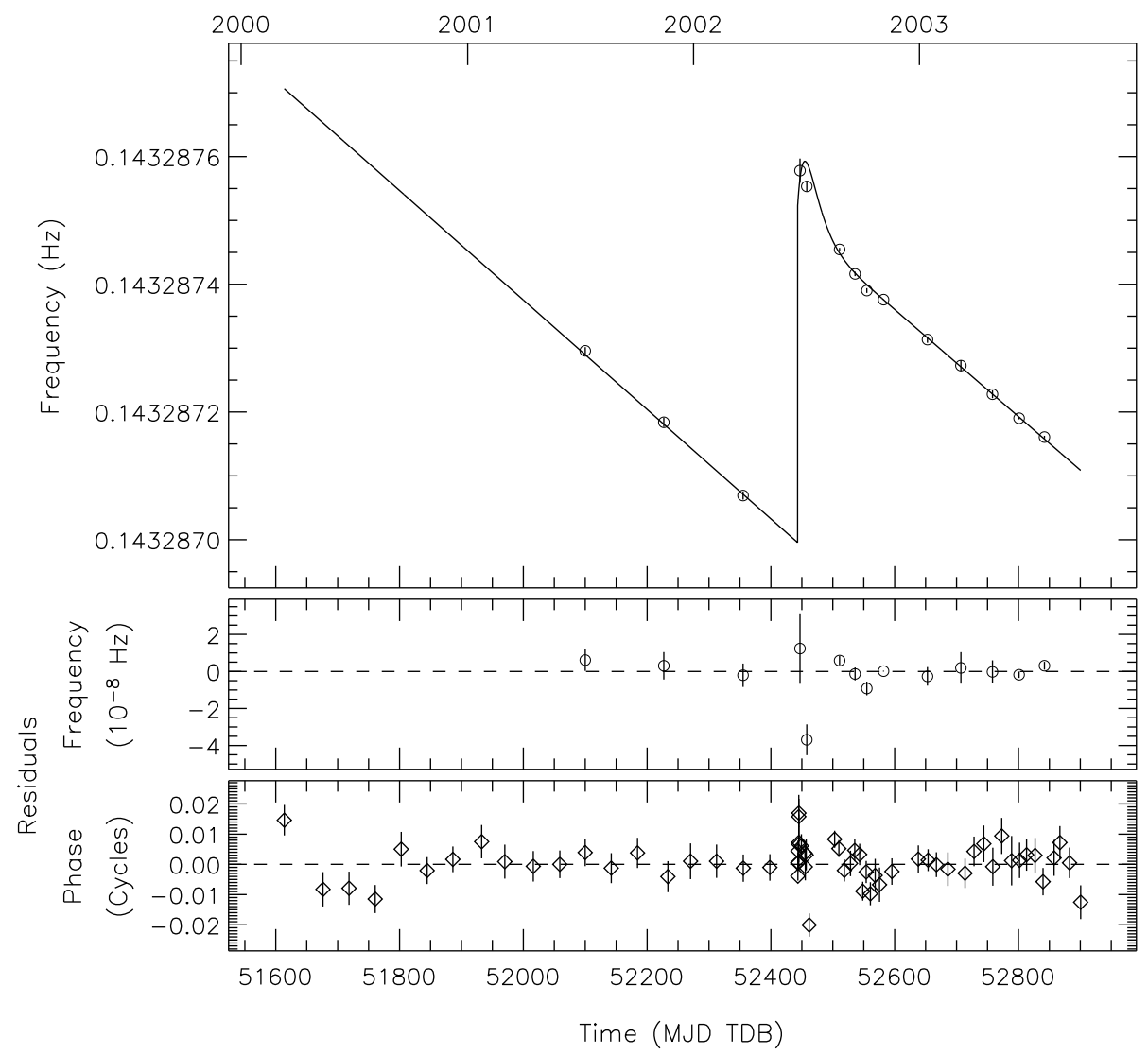

Fig. 7. - Top Panel - Frequency evolution of 1E 2259+586 around the time of the outburst for a model including an exponential rise and fall in frequency post-glitch. See text for details. The solid line represents the best-fit model (see Table 4 for model parameters). The circles denote frequency measurements for independent subsets of data. The effect of the glitch is obvious, as is the partial recovery. Middle Panel-The frequency residuals of the independent frequency points minus the model. Bottom Panel-Phase residuals with respect to the best-fit model. Closer inspection of the residual cluster just following the outburst epoch reveals that there is a low amplitude systematic trend. This is discussed further in the text.

The parameters in Table 4 show that the glitch consisted of a total fractional frequency increase $\Delta \nu_{\max } / \nu=(4.24 \pm 0.11) \times 10^{-6}$ where $\Delta \nu_{\max }$ is the maximum upward excursion in frequency relative to the pre-glitch ephemeris. Of this frequency jump, a fraction $Q \equiv\left(\Delta \nu_{\max }-\Delta \nu-\Delta \nu_{g}+\Delta \nu_{d}\right) / \Delta \nu_{\max }=0.185 \pm 0.010$ recovered within two months following 
the glitch.

We note that a decrease in the magnitude of the spin-down rate would be unique among all known pulsar and AXP glitches (Shemar \& Lyne 1996; Kaspi \& Gavriil 2003). This is discussed more in $§ 4.2 .5$. Alternatively, some radio pulsars have shown a long-term exponential rise in frequency post-glitch. However, our best fit to this model was excluded by the frequency data.

The exponential rise term in this frequency model is clearly preferred by the data, however, several important caveats must be stated. The exponential form was chosen due to its success in modeling radio pulsar glitches (Lyne et al. 1993). However, for the 19 days following the glitch, there is a significant deviation from this model that constitutes a large portion of the remaining $\chi^{2}$ in the fit. During this 19 day interval, the best fit model predicts rapid spin up for the first $\sim 10$ days after which the frequency derivative again becomes negative. If we fit only the data from the 12 days following the glitch, we measure a frequency derivative of $-1.0 \pm 0.3 \times 10^{-13} \mathrm{~Hz}$

$\mathrm{s}^{-1}$ whereas the model predicts an average frequency derivative of $+5 \times 10^{-14} \mathrm{~Hz} \mathrm{~s}^{-1}$. Summing the frequency derivative measurement error and the model error in quadrature, we find that the two values differ at the $\sim 5 \sigma$ level. Hence, there is no direct proof that there was significant spin up during the first 12 days following the glitch. Note also that the frequency derivative measurement is only at the $3 \sigma$ level, so some spin up cannot be completely ruled out. Another caveat when considering the exponential rise in frequency is the pulse profile was undergoing large changes during this same time interval (see $\S 3.4$ ), thus comprimising our ability to phase align with our template pulse profile. In particular, if the pulse profile was shifting in phase in a smooth manner as it changed shape, then this shift in phase would manifest itself as an apparent change in frequency. It is not likely that the deviation from a pure exponential recovery can be attributed entirely to a systematic shift in the pulse profile, as that would require a large drift of $\sim 0.35$ cycles within the two weeks following the glitch. Furthermore, even if we exclude from our fit the data in which the pulse profile changes were largest, the growth term still significantly improves the fit over the simple exponential recovery $\left(\Delta \chi^{2}=136\right.$ for 2 fewer dof $)$. We conclude from our analysis that there is a significant deviation from a simple exponential recovery, however, due to gaps in the data coverage (in particular from 19 to 60 days after the glitch) and the inherent pulse profile changes, we could not precisely identify the manner in which the frequency evolution deviated.

\subsection{Pulsed Flux History}

Coincident with the burst activity on 2002 June 18 was a sudden increase in the pulsed flux from 1E 2259+586 (Kaspi et al. 2003). The pulsed intensity of the AXP decreased through the burst observation as did the burst rate, thereby confirming the AXP as the origin of the burst emission. Here, we put the pulsed flux enhancement in context with the long-term pulsed flux history and track the recovery back toward the pre-outburst level.

Using the ephemeris determined in the last section, pulse profiles of the PCA data $(2-10$ 
$\mathrm{keV}$ ) from 2000 March through 2003 June were constructed. The pulse profiles were generated for each pointing at times before and up to one year following the outburst. For the 2002 June 18 observation, the bursts were removed and the data were split into $\sim 200 \mathrm{~s}$ segments before folding. Between 1 day and 19 days after the outburst, the data were grouped by spacecraft orbit ( $3 \mathrm{ks})$. Once the pulse profiles were constructed, the pulsed intensity was measured by first decomposing each pulse profile in terms of its Fourier powers. The power in the first 7 harmonics was summed to give the RMS pulsed intensity (see Eq. 1). The pulsed flux history of 1E 2259+586 is shown in Figure 8. The large spike indicates the time of the outburst. Note that the pulsed flux has not yet returned to the pre-outburst level. The time scale and functional form of the recovery is studied in more detail in $\S 3.6$.

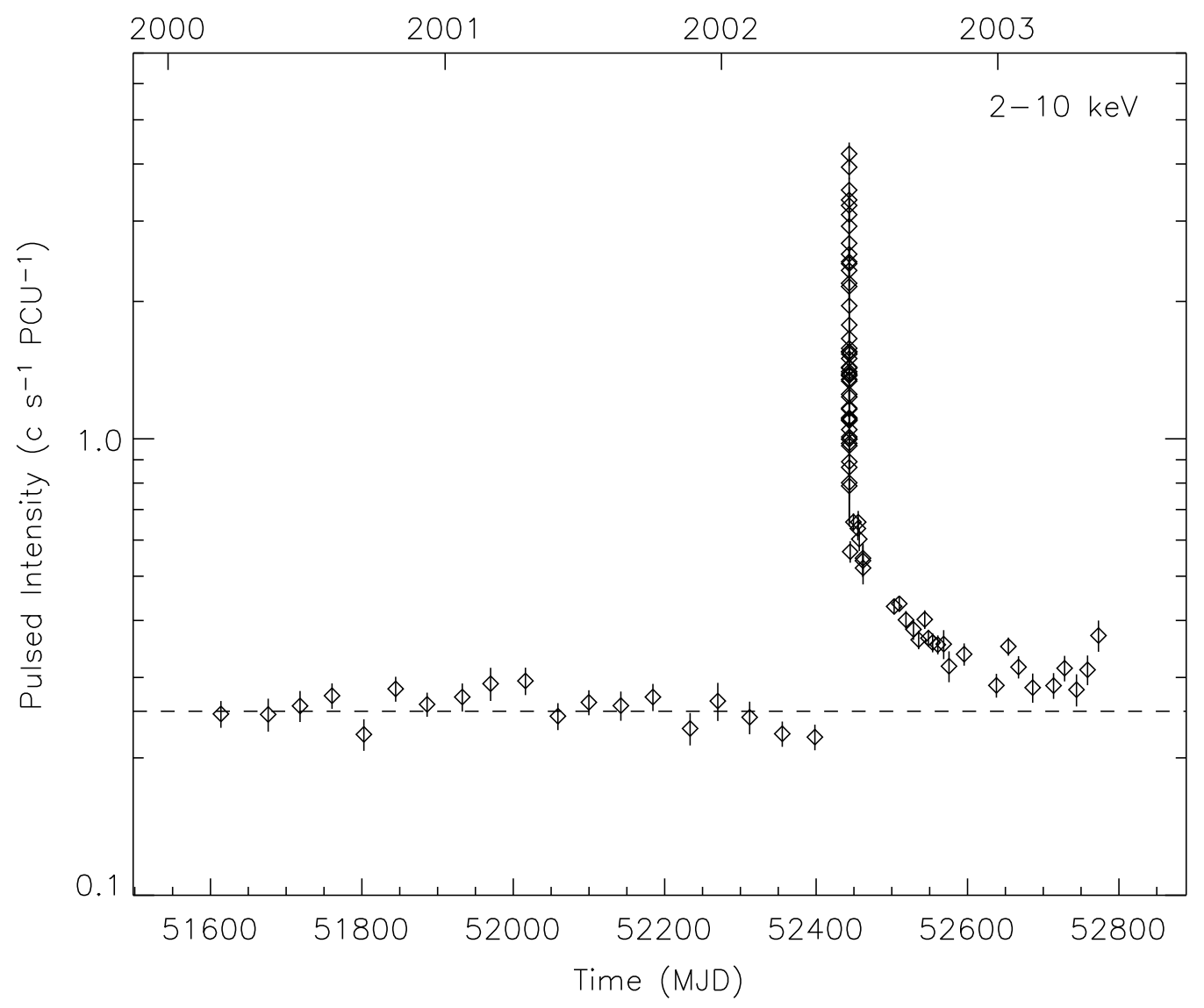

Fig. 8. - The pulsed flux history of 1E 2259+586 (2-10 keV) from 2000 March through 2003 June as measured using the $R X T E$ PCA. The sharp rise in the pulsed intensity coincides with the burst activity. 


\subsection{Pulsed Fraction}

The PCA is a sensitive X-ray detector with a wide FOV and no imaging capabilities (for a fixed pointing). Due to the design of the instrument, it is not possible to reliably measure the pulsed fraction and/or spectrum of weak X-ray sources such as $1 \mathrm{E} 2259+586$ on account of uncertainties in the background, particularly in the direction of the Galactic plane. However, during episodes where the AXP flux increases substantially, one can measure accurately the pulsed fraction and spectrum (see §3.5) with the PCA under certain assumptions (Lenters et al. 2003).

The observed count rate in the PCA during pointings of 1E 2259+586 consists of several components, namely the central source, instrumental background, near-Earth background due to precipitating particles, the "diffuse" cosmic background, the Galactic ridge, the bright SNR surrounding the AXP, and other dim sources in the FOV. To disentangle the spectrum of the central source from all the other contributions to the net count rate, the remaining components must somehow be modeled. The instrumental and near-Earth background (i.e. local background) can be subtracted using housekeeping data and models provided by the $R X T E$ team $^{11}$. Fortunately, the remaining components do not vary greatly on time scales of months to years.

To estimate the count rate in the PCA of the remaining background components, we did the following. Using the last PCA observation (2002 May 04) taken before the outburst, we selected counts in the range 2-10 keV from all PCUs other than PCU0 and subtracted the background as estimated using the FTOOL PCABACKEST with the combined model (CM) version of 2003 March 30. Note that PCU0 was excluded due to the loss of the propane layer and the resulting large increase in the magnitude and variance of the background rate. Next, we measured the pulsed count rate (per PCU) during this observation. Assuming that the $2-10 \mathrm{keV}$ pulse fraction measured during the XMM-Newton observation from 2002 June 11 (Table 2) was the same on 2002 May 04, we determined the background rate in the PCA. We use this "cosmic" background rate for all observations during and following the outburst where we measure the pulsed fraction. In using this count rate for our background, we assume that $(i)$ the AXP pulse fraction was the same on 2002 May 04 as it was on 2002 June 11 and (ii) the cosmic background remains constant from 2002 May 04 until 2002 July 06 (the time of the last pulsed fraction measurement reported here).

Using this technique, we determined that the pulsed fraction of $1 \mathrm{E} 2259+586$ decreased relative to the pre-outburst level while the source was burst active (Kaspi et al. 2003). Here, we have extended this analysis to later PCA observations through 2002 July 06. As with the $X M M$-Newton data, we measured the pulsed fraction by subtracting the background and decomposing the folded pulse profile in terms of its Fourier powers. The RMS pulsed fraction was determined from the sum of the first 7 harmonics using the formalism described in $\S 2.1$. The $R X T E$ PCA pulsed fraction measurements are plotted along with the XMM-Newton measurements (Table 2) in Figure 9. We found that the $2-10 \mathrm{keV}$ pulsed fraction decreased initially to a level of

\footnotetext{
${ }^{11} \mathrm{ftp}$ ///legacy.gsfc.nasa.gov/xte/calib_data/pca_bkgd/
} 
$\sim 15 \%$ where it remained for at least one day before rapidly returning to the pre-outburst value within $\sim 6$ days of the outburst.

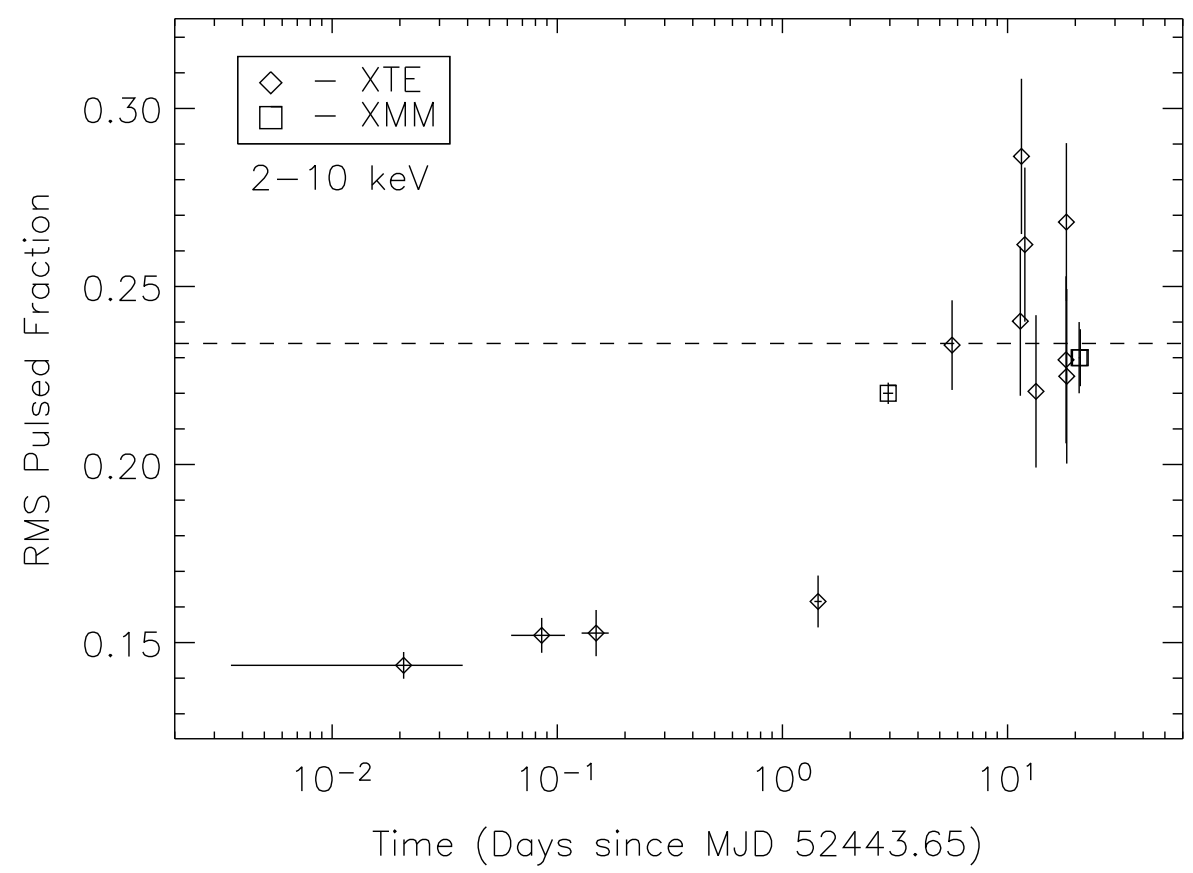

Fig. 9. - The time evolution of the $2-10 \mathrm{keV}$ pulsed fraction of $1 \mathrm{E} 2259+586$ through the outburst of 2002. Diamonds denote measurements made with the RXTE PCA and squares mark XMMNewton measurements (Table 3). The horizontal dashed line marks the pulsed fraction from the $X M M$-Newton observation one week prior to the outburst (Obs2).

\subsection{Pulse Profile}

Coincident with the detected burst activity in the PCA from 1E 2259+586 was a sudden change in the folded pulse profile (Kaspi et al. 2003). In the energy range $2-5 \mathrm{keV}$, the relative amplitudes of the two peaks were switched while the source was burst active. In this energy range, the pulse profile returned to near its pre-outburst form within $\sim 6$ days. No changes like this have been seen in more than 6 years of monthly monitoring with the PCA (Gavriil \& Kaspi 2002), although similar changes have been noted prior to the PCA monitoring (Iwasawa, Koyama \& Halpern 1992). Here, we investigate further the pulse profile evolution over a longer time baseline and as a function of energy.

The temporal evolution of the folded pulse profile of 1E $2259+586$ from $2-10 \mathrm{keV}$ is shown in Figure 10. From the pulsed flux analysis, we know that the amplitudes of both peaks increased 
during the 2002 June 18 observation relative to their pre-outburst amplitudes. Furthermore, the pulsed flux decreased significantly during the burst observation (RXTE orbits $1-3$ ). This allows

us to conclude that the amplitude of peak 1 is decaying more rapidly than peak 2 during the burst observation (as opposed to peak 2 growing relative to peak 1). The variability in the 18 days following the burst activity (panels 5-8) shows erratic behavior in the pulse morphology. It is not until several weeks after the outburst that the pulse profile closely resembles its pre-outburst form within this energy range. Even at several months following the outburst the differences are significant. Specifically, the bridge of emission connecting the two peaks is higher than it was pre-outburst.

We quantified the change in pulse shape $(2-10 \mathrm{keV})$ by decomposing the pulse profile in terms of its Fourier powers following Equation 1. As is clear from Figure 11, the ratio of the power in the second harmonic to the first harmonic (i.e. fundamental frequency) is the dominant factor governing the pulse shape change. During quiescence, the second harmonic contains more than $80 \%$ of the total power. During the observation containing the bursts, the power in the first harmonic increased such that there is actually as much or more power in this harmonic compared with the second harmonic. The ratio of the power in the first two harmonics recovers gradually over the next several months.

Lastly, we investigated the dependence of the pulse shape on photon energy during the burst observation when the pulse shape was distorted the greatest. We find that there is a significant energy dependence of the pulse shape that becomes more prominent toward the end of the burst observation. Specifically, peak 2 becomes narrower at high energies and the centroid of this peak lags in phase by $\sim 0.02$ cycles $(0.15 \mathrm{~s})$ from 2 to $15 \mathrm{keV}$. Both the centroid and phase of peak 1 are consistent with remaining unchanged versus energy. Similarly, the relative amplitudes of peak 1 and peak 2 are consistent with being constant in this energy range.

\subsection{Spectroscopy}

During the outburst detected on 2002 June 18, the X-ray spectrum of 1E 2259+586 became much harder (Kaspi et al. 2003). Initially, the blackbody temperature increased to $\sim 1.7 \mathrm{keV}$ (from $\sim 0.42 \mathrm{keV}$ ) and the photon index flattened to $\sim 2.5$ (from $\sim 4.2$ ). During the next 4 hours, the spectrum of $1 \mathrm{E} 2259+586$ evolved back toward its quiescent shape, but did not recover fully. Here, we analyze the RXTE PCA observations that took place over the next few weeks to track the recovery of the AXP spectrum.

As with the pulsed fraction, measuring the X-ray spectrum of $1 \mathrm{E} 2259+586$ with the $R X T E$ PCA is not straightforward, so we used a similar technique in subtracting the background as that employed to measure the pulse fraction (see §3.3). As before, the last PCA observation carried out on 2002 May 04, 46 days before the outburst, was used to estimate the cosmic background in the PCA FOV. First, the local background during this observation was estimated using the FTOOL 

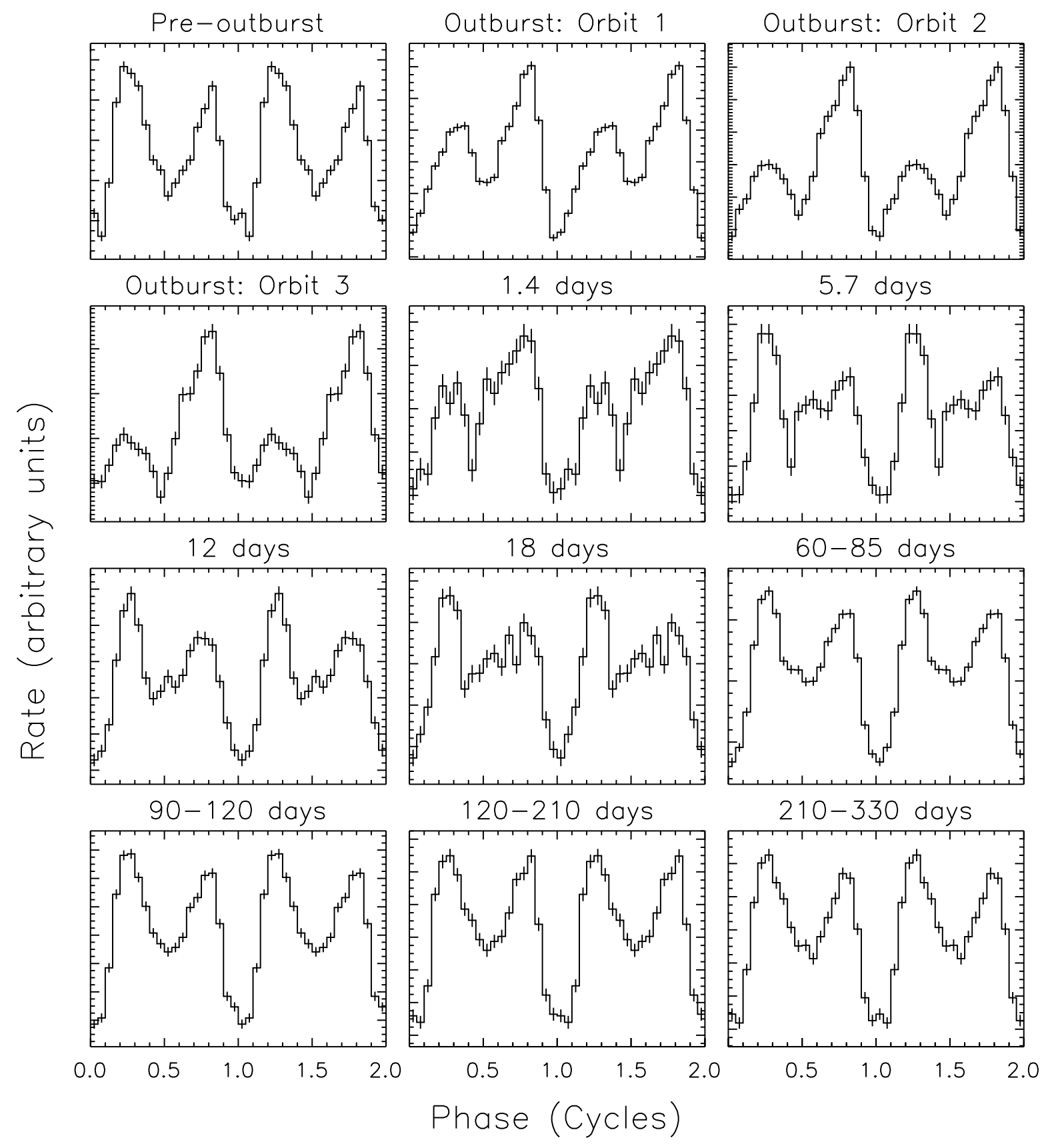

Outburst: Orbit 2

Fig. 10. - The time evolution of the $2-10 \mathrm{keV}$ pulse profile of $1 \mathrm{E} 2259+586$ through the outburst of 2002. Profiles have arbitrary flux units and two pulse cycles are shown for clarity. Time increases from left to right and top to bottom. 

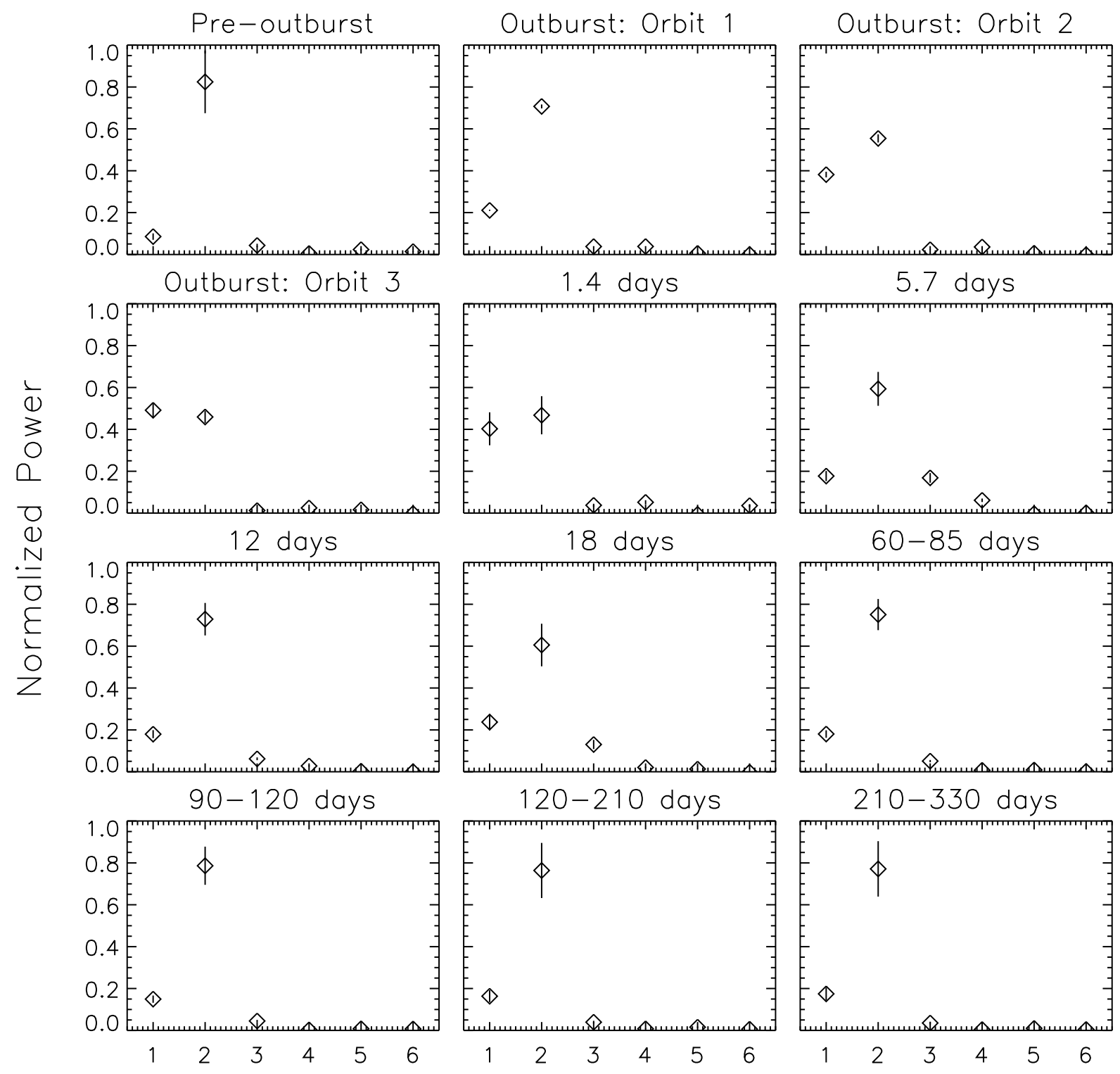

Harmonic Number

Fig. 11. - The time evolution of the Fourier power harmonic distribution of the $2-10 \mathrm{keV}$ pulse profile of $1 \mathrm{E} 2259+586$ through the outburst of 2002. Power levels have been normalized to the total power of the first 7 harmonics. Time increases from left to right and top to bottom. 
PCABACKEST and the combined model of 2003 March 30. The observed Standard 2 spectrum $^{12}$ was grouped such that there were at least 25 total counts per bin (source plus background) and then fit (less the local background) using XSPEC v11.2.0 to a multi-component model. The model consisted of the standard AXP spectrum plus another blackbody and a Gaussian line. The second blackbody component and Gaussian line were each modified by interstellar absorption with a fixed column density $\left(N_{H}=2 \times 10^{22} \mathrm{~cm}^{-2}\right)$. The AXP spectral parameters were frozen to the values obtained from the XMM-Newton observation of 2002 June 11 (see Table 3), 39 days following this observation and 7 days prior to the outburst. Using this model, we obtained a good fit to the PCA data between 2.5 and $20.0 \mathrm{keV}\left(\chi_{\nu}^{2}=0.71\right.$ for $\left.35 \mathrm{dof}\right)$. The free components in this fit (i.e. blackbody and Gaussian line), therefore, define the spectrum of the remaining background in the PCA for this particular pointing at this particular epoch. This model was used to define the cosmic background in the PCA detector during and directly following the burst activity was used assuming that these components (e.g. Galactic ridge, SNR, etc.) remain constant between 2002 May 04 and 2002 July 06.

Standard 2 spectra were accumulated for seven time intervals during the 2002 June 18 observation when the bursts were detected. Note that the bursts themselves were eliminated from the data used to create the spectra. Spectra were also accumulated for each PCA observation from 2002 June 20 through July 06. Fit results from the 2002 June 18 observation showed that the standard AXP spectral model (blackbody plus power law) best fit the data (Kaspi et al. 2003). We fit the same model to the subsequent PCA observations (2.5-20.0 keV) through 2002 July 06. The RXTE fit results are shown in Figure 12 along with the XMM-Newton measurements given in Table 3.

The unabsorbed flux decayed rapidly within the first day of the outburst. The functional form of the decay depends critically upon the reference epoch chosen. Both power-law and exponential decay models yield acceptable fits to the data. At 20 days following the outburst, the AXP is still a factor of $\sim 2$ brighter than its nominal (quiescent) flux level. The flux decay is covered in greater detail in the next section. The remaining spectral parameters shown here recover to within $25 \%$ of their pre-burst levels within the first $\sim 1-3$ days. Using the pulsed flux history as an indicator for the recovery time scale, a full recovery of the spectral parameters would not be expected until $\sim 1$ year later. However, variation in these spectral parameters at the $\sim 25 \%$ level has been seen outside of burst activity (see $\S 2.2$ ), thus these parameters may not decay further.

It is interesting to note that the X-ray spectrum of $1 \mathrm{E} 2259+586$ was harder when the spindown rate was higher during the first month following the glitch. This behavior in $1 \mathrm{E} 2259+586$ is qualitatively consistent with the spectral hardness versus spin-down rate correlation found by Marsden \& White (2001) who considered the AXPs and SGRs as a whole. This suggests that individual members of this class that have shown significant variability in spin-down rate may also show correlated spectral variations.

\footnotetext{
${ }^{12}$ As with the pulsed fraction analysis, all data from PCU0 were excluded from the spectral analysis.
} 

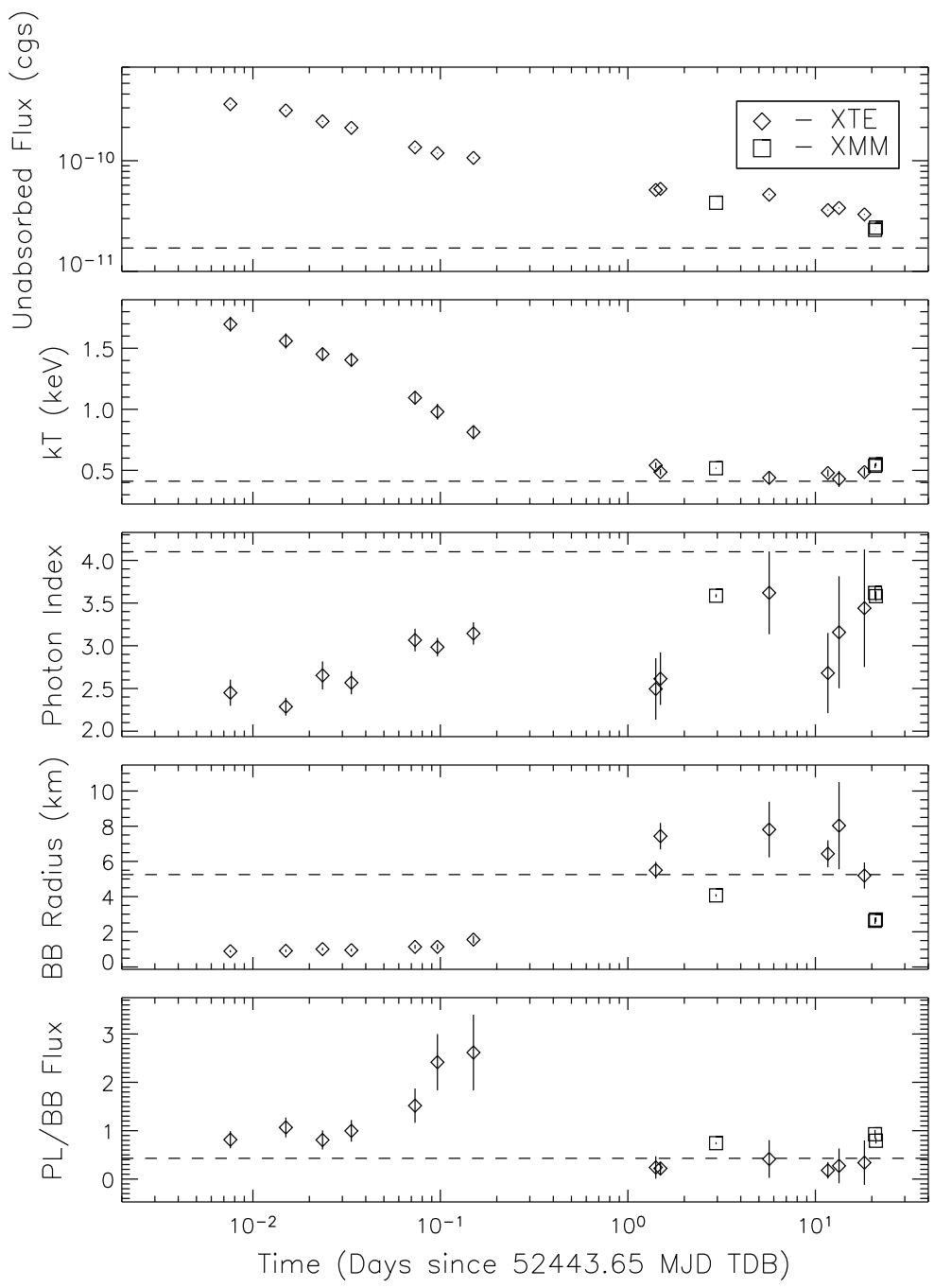

Fig. 12. - The spectral evolution of 1E $2259+586$ through and following the outburst of 2002. From the top panel down: the unabsorbed flux $(2-10 \mathrm{keV})$, the blackbody temperature $(k T)$, the photon index, the blackbody radius, and the ratio of power-law $(2-10 \mathrm{keV})$ to bolometric blackbody flux. A distance of $3 \mathrm{kpc}$ was assumed (Kothes, Uyaniker, \& Aylin 2002) to compute the blackbody radius. Horizontal dashed lines denote the values of each parameter during the XMM-Newton observation one week prior to the outburst.

We note that the later RXTE PCA spectral measurements ( $>1$ day) agree reasonably well with the XMM-Newton measurements contained within this interval, however, they are systematically offset from one another. This could be due to systematic effects in our background 
subtraction method and/or a deviation in the power-law spectral model at high energies where the two instrumental responses do not overlap (12-20 keV).

\subsection{Flux Decay and Energetics}

To better quantify the flux decay of $1 \mathrm{E} 2259+586$ following the outburst of 2002 June, we combined the XMM-Newton flux measurements with our RXTE PCA pulsed flux measurements which span a much broader temporal baseline. The advantage of using the PCA pulsed flux measurements as opposed to the phase-averaged flux measurements is that the systematic errors present in the background subtraction are not a concern.

The pulsed flux measurements were converted to unabsorbed phase-averaged fluxes in the following way. First, we determined a conversion factor between the two by calculating the ratio of the pre-outburst unabsorbed flux measured with $X M M$-Newton to the average PCA pulsed flux for the year leading up to the outburst. Assuming that the pulsed fraction and spectrum do not change, this factor can be multiplied with subsequent PCA pulsed flux values to estimate the unabsorbed phase-averaged flux at those times. However, we know that both the pulsed fraction and the energy spectrum changed during this outburst. We corrected for the brief period where the pulsed fraction decreased by multiplying by an additional factor of the ratio of the nominal pulsed fraction to the observed pulsed fraction at those times. This factor was only applied to the PCA pulsed flux measurements within the first 2 days following the burst activity where the fraction dropped from $23 \%$ to $\sim 15 \%$. Computing this conversion factor for a broad energy range $(2-10 \mathrm{keV})$ reduces the effects of spectral changes. In fact, when we applied our pulsed flux to unabsorbed phase-averaged flux conversion factor to the data where we have independent unabsorbed flux measurements at early times in the outburst ( $<20$ days after burst activity), the agreement between the two measurements was found to be quite good (Figure 13). Since the spectral differences are greatest at these times in the outburst, we feel that this technique is a robust one for constructing a long-term light curve for the source.

In this way, we have taken the pulsed flux measurements presented in Figure 8 and converted them to phase-averaged unabsorbed flux values. These data are plotted relative to the glitch epoch (Table 4) in Figure 13 in addition to the three XMM-Newton flux measurements. Clearly, the flux decay is not well described by a single component model (e.g. exponential or power law). The temporal decay of the flux during the PCA observations containing the burst activity $(<1$ day) is much more rapid than the decay during the year following the burst activity. We split the data into two segments ( $<1$ day and $>1$ day after the glitch), and fit each independently to a power-law model $\left(F \propto t^{\alpha}\right)$. The measured temporal decay indices for the two segments are -4.8 \pm 0.5 and $-0.22 \pm 0.01$, respectively.

The RXTE All-Sky Monitor (ASM) observed 1E 2259+586 at 03:50 and 14:43 UT on 2002 June 18, before the observed burst activity and following the measured glitch epoch, although 


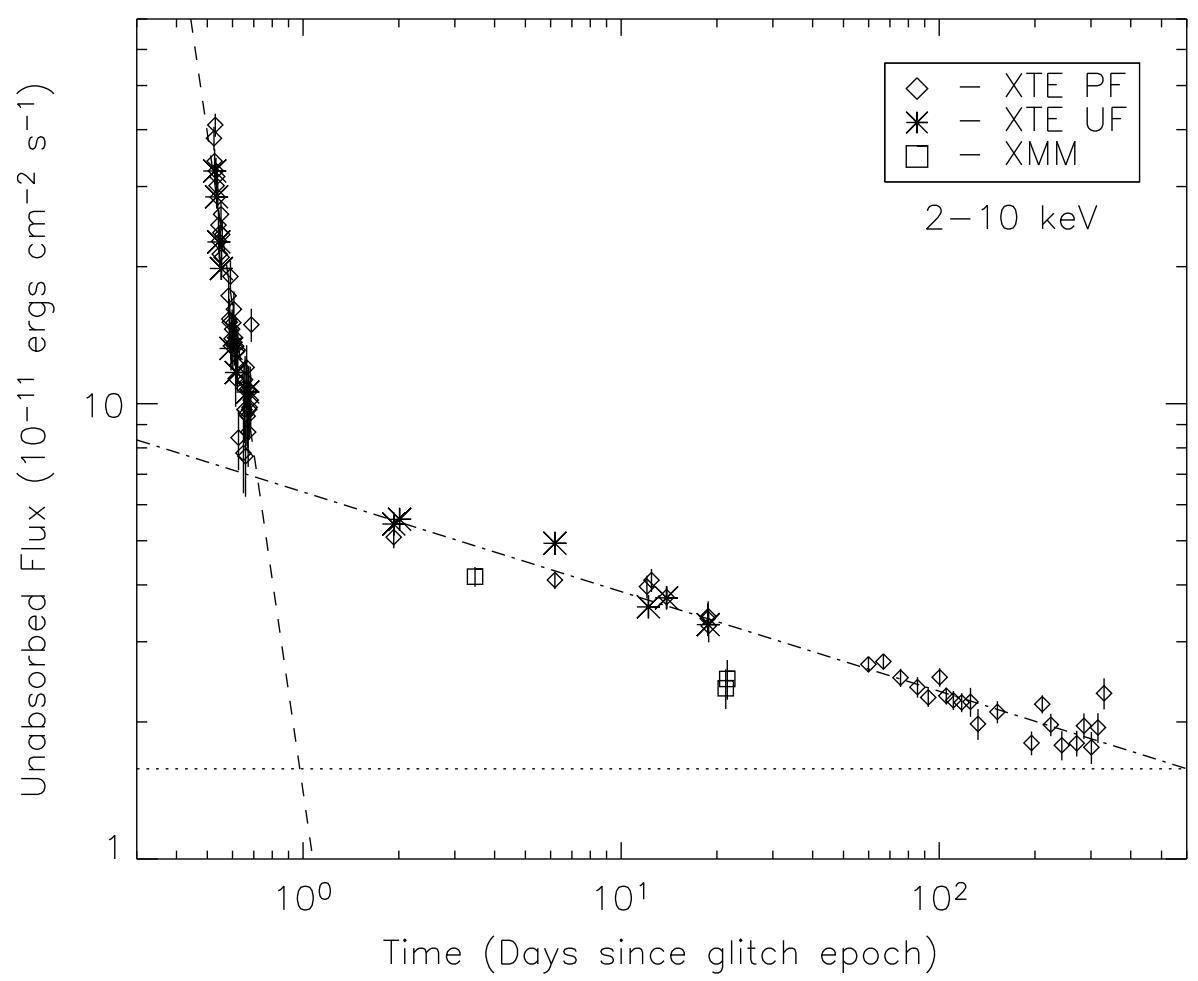

Fig. 13.- The time evolution of the unabsorbed flux from 1E 2259+586 following the 2002 June outburst. The glitch epoch (Table 5) is used as the reference time for this plot. Diamonds denote inferred unabsorbed flux values calculated from $R X T E$ PCA pulsed flux measurements. Asterisks and squares mark independent phase-averaged unabsorbed flux values from $R X T E$ and $X M M$ Newton, respectively. The dotted line denotes the flux level measured using XMM-Newton 1 week prior to the glitch. The dashed line is a power-law fit to the PCA flux measurements during the observations containing the burst activity ( $<1$ day). The dot-dash line marks the power-law fit to all data $>1$ day following the glitch. See text for further details.

the first observation is within the errors of the measured glitch epoch. If we extrapolate the slower flux decay model $(\alpha=-0.22)$ back to the time of the ASM observations, we find that the expected flux values (14 and $8 \times 10^{-11} \mathrm{ergs} \mathrm{cm}^{-2} \mathrm{~s}^{-1}$, respectively) are comfortably below the $99 \%$ confidence upper limit of $1 \times 10^{-9} \mathrm{ergs} \mathrm{cm}^{-2} \mathrm{~s}^{-1}$ (Kaspi et al. 2003), thus the ASM limits are not constraining for this component. For the steeper component $(\alpha=-4.8)$ containing the burst activity, we find that the expected flux at the time of the first ASM observation is several orders of magnitude above the upper limit. The second ASM observation (52443.611 MJD TDB) is unconstraining. This suggests that the onset of burst activity (associated with the first decay component) must have come after the earlier ASM observation (52443.158 MJD TDB) or the 
flux enhancement deviated from this steep decay between the glitch epoch and the $R X T E$ PCA observations. One possibility is that the onset of this burst activity was delayed with respect to the glitch epoch. In fact, a much later onset time for the burst activity is inferred from the time evolution of the burst recurrence frequency (Gavriil et al. 2003).

The absence of a fiducial point for the initial, rapid flux decay associated with the burst activity does not allow us to accurately measure the temporal decay index of this component. Choosing a reference epoch just before the time of the first bursts detected with the PCA yields a decay index less than unity (in magnitude). Thus, we can only constrain the index of the flux decay within the time span containing the burst activity to be $<6.6$ (in magnitude). Note that as the reference epoch approaches the start of the burst observation, the flux decay becomes steeper than a power law in form (e.g. exponential). This is discussed further in $§ 4.1 .1$. Unlike the early component, the temporal index of the more gradual flux decay in the months following the glitch ( $\alpha=-0.22$ ) is insensitive to varying the reference epoch between the time of the glitch and the beginning of the observed burst activity.

We next used these power-law fits for estimating the total energy released during this outburst. We integrated each model, less the quiescent flux level (Figure 13), only over the time ranges where we have flux measurements (i.e. from the start time of the burst observation onward). In spite of the large range of allowed temporal decay indices, the energy we measure in the early flux decay component is well determined since we integrate the model only over the interval where we have observations (i.e. we do not extrapolate the model back toward the glitch

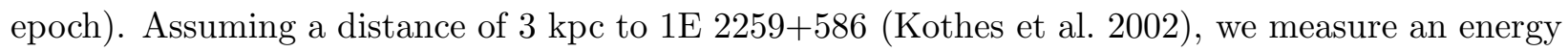
release $(2-10 \mathrm{keV})$ of $2.7 \times 10^{39} \mathrm{ergs}$ and $2.1 \times 10^{41} \mathrm{ergs}$ for the fast and slow decay intervals, respectively. In terms of the overall energy budget, the energy released in the bursts themselves $\left(6 \times 10^{37}\right.$ ergs over $2-60 \mathrm{keV}$ [Gavriil et al. 2003] $)$ is insignificant in comparison to the excess persistent emission in X-rays released during the year following the outburst. Moreover, the excess persistent flux emitted during the interval containing the burst activity is insignificant in comparison to the total energy released during the year-long flux decay.

\section{Discussion}

Virtually all measurable X-ray properties of 1E 2259+586 changed suddenly and dramatically during the 2002 June outburst. Continued observations with RXTE and XMM-Newton have allowed us to track the recovery of several source parameters shown to change during this outburst (Kaspi et al. 2003) and identify additional parameters that were similarly affected. Many of the observed variations resemble phenomena seen in other classes of neutron stars, namely SGRs and radio pulsars. Here, we compare the AXP outburst properties with similar phenomena seen in those source classes in the hopes of identifying similarities and differences that can help elucidate the physical properties of these different manifestations of young neutron stars. Specifically, we consider $(i)$ the radiative properties of the persistent and pulsed emission during and following 
the outburst and compare these with those seen in SGRs, since that is the only other source class for which such outbursts have been seen and (ii) the rotational behavior of the pulsar and compare it both with behavior seen in radio pulsars as well as in SGRs and in another AXP, 1RXS J1708-4009. Finally, we point out that the detection of low-intensity outbursts in AXPs has important implications for our estimates of the number of active magnetar candidates in our Galaxy. The 1E 2259+586 burst properties and their relation to SGR burst properties are considered separately (Gavriil et al. 2003)

\subsection{Transient X-ray Emission and Pulse Properties}

We have shown that there are two components to the flux decay in $1 \mathrm{E} 2259+586$ during the 2002 June outburst. There is a rapid decay of the flux during the observations containing the burst activity and then there is a more gradual flux decay seen in the year following the glitch. During the initial flux decay, the spectrum was considerably harder than at all other times while the spectral hardness at times $>1$ day after the glitch are consistent with pre-glitch spectral measurements. The spectral differences in the two decay components (total energy, blackbody radius, etc.) point toward separate physical mechanisms for the two flux enhancements (see Figures 9 and 12).

Coincident with the glitch and burst activity was a dramatic change in the pulse profile of $1 \mathrm{E} 2259+586$. The majority of the observed shape change recovered within $\sim 6$ days, but there was still some residual change that slowly decayed over the months following the outburst (§3.4). The 2-10 keV pulsed fraction dropped to $~ 15 \%$ during the observed burst activity and quickly recovered to the pre-outburst level of $23.4 \%$ within $\sim 6$ days $(§ 3.3)$.

Here, we compare the observed properties of the flux enhancement and pulse properties in 1E 2259+586 to qualitatively similar behavior detected in SGRs and briefly discuss how the changes seen in $1 \mathrm{E} 2259+586$ can be accomodated within the magnetar model.

\subsubsection{Comparison to SGR Outbursts: X-ray Flux and Spectrum}

The richest SGR database with which to make an empirical comparison to the 1E 2259+586 outburst comes from the most active SGR during the last 6 years, SGR 1900+14. This SGR has been observed on 14 separate occasions since 1997 by imaging X-ray telescopes and more than 100 times with RXTE. Within this time span, SGR 1900+14 entered several burst active episodes; the most notable of which was the outburst that began on 1998 August 27 with a giant flare having a total energy $\sim 10^{44}$ ergs (e.g. Feroci et al. 2001). Coincident with this giant flare was a large increase in the persistent and pulsed flux from the source (e.g. Woods et al. 2001), in addition to a dramatic change in the pulse profile and a timing anomaly (see $\S 4.2 .3$ ). 
A spectral analysis of the RXTE PCA observation of SGR 1900+14 one day following the giant flare shows that the blackbody temperature was higher than the nominal temperature $(\sim 0.5$ $\mathrm{keV}$ ) at $0.94 \mathrm{keV}$ (Woods 2003). Two other high fluence bursts from SGR 1900+14 have extended X-ray tails or afterglows that show enhanced thermal emission at times reaching up to $4 \mathrm{keV}$ (Ibrahim et al. 2001; Lenters et al. 2003). The thermal component of 1E 2259+586 shows a similar brightening and temporal decay. Here, the temperature rose from $0.4 \mathrm{keV}$ up to $1.7 \mathrm{keV}$ at the onset of the outburst before quickly decaying to $0.5 \mathrm{keV}$ within the first few days. Accompanying the temperature increase in $1 \mathrm{E} 2259+586$ was a significant hardening of the photon index. This is different than what has been seen in SGR 1900+14 where there has been either no change in photon index after some bursts (Lenters et al. 2003) or even a slight softening of the non-thermal component of the spectrum (Woods 2003).

An analysis of four separate bursts/flares from SGR 1900+14 and their associated afterglows (Lenters et al. 2003) shows that the emitted energy within the afterglow (2-10 keV) corresponds to roughly $2 \%$ of the burst energy $(25-100 \mathrm{keV})$. Due to the bandpass of the PCA, we have only been able to measure the burst energy within the $2-60 \mathrm{keV}$ range (Gavriil et al. 2003). However, we can estimate the burst energy $(25-100 \mathrm{keV})$ during the 2002 June outburst by taking the measured count fluence with the PCA (Gavriil et al. 2003) and multiplying by the counts-to-energy conversion factor determined for SGR 1900+14 (Gögü et al. 1999). This conversion factor was used because the mean burst spectral hardness in the PCA was similar for 1E 2259+586 (Gavriil et al. 2003) and SGR 1900+14 (Göğüş et al. 2001). The total energy was further modified by a factor 2 increase to roughly compensate for gaps in the data due to Earth occultation during the PCA pointing. Assuming that the energy spectra of the $1 \mathrm{E} 2259+586$ bursts above $60 \mathrm{keV}$ is similar to SGR $1900+14$, we estimated the burst energy released by $1 \mathrm{E} 2259+586$ to be $\sim 1 \times 10^{38}$ ergs $(25-100 \mathrm{keV})$. The brightening of $1 \mathrm{E} 2259+586$ during the 2002 June outburst contained two components: a rapid decay during the initial burst observation followed by a more gradual flux decay in the year following the glitch. The energy released from 1E 2259+586 within these two components is $\sim 30$ and $\sim 2000$ times greater than the burst energy (Table 5) - in stark contrast to the value of 0.02 found for SGR $1900+14$. However, we note that a long duration, low-peak-flux burst preceding the RXTE PCA observation on 2002 June 18 cannot be excluded as a possible trigger for the early flux enhancement. If we assume the $2 \%$ ratio of afterglow to burst energy observed in SGR 1900+14 and we apply the gamma-ray flux $(15-150 \mathrm{keV})$ upper limit of $1 \times 10^{-6}$ ergs $\mathrm{cm}^{-2} \mathrm{~s}^{-1}$ from the Ulysses gamma-ray burst detector (K. Hurley, private communication), the duration of the putative burst must be $\gtrsim 300$ s to yield $1.5 \times 10^{41}$ ergs in burst energy.

The temporal decay of the flux from SGR 1900+14 following the August 27 flare follows a power law in time with an exponent -0.71 during the 40 days following the flare (Woods et al. 2001). For a power-law fit to the flux evolution during the burst activity, we can only constrain the decay to have an exponent less than 6.6 (in magnitude) due to the uncertainty in the reference epoch (see $\S 3.6$ ). Thus this decay component is not inconsistent with a -0.7 power law. The more gradual flux decay in the months following the glitch obeyed a power law in time that scaled 
as $t^{-0.22 \pm 0.01}$, significantly flatter than the decay following the August 27 flare or any other flux decay following bright bursts from SGR 1900+14 (Lenters et al. 2003; Feroci et al. 2003).

We can also compare the energetics and flux decay of $1 \mathrm{E} 2259+586$ to those seen in SGR 1627-41. This SGR has shown one outburst in 1998 where $98 \%$ of the emitted burst energy was concentrated into a narrow 3-week window (Woods et al. 1999a). The flux from the source decayed over the next 3 years approximately as a power law in time with an exponent -0.47 (Kouveliotou et al. 2003). The last two Chandra observations of this SGR show that the flux has leveled out (at least temporarily). Since there are no pre-outburst flux measurements to establish the "quiescent" flux level of this SGR, we take the two latest flux measurements as the quiescent flux level. Under these assumptions, the burst tail or afterglow energy during the four years following the 1998 outburst is comparable to the energy released in the bursts themselves (Kouveliotou et al. 2003).

Table 5: SGR and AXP burst/afterglow energetics ${ }^{a}$ and temporal decay indices.

\begin{tabular}{ccccc}
\hline \hline & SGR 1900+14 & SGR 1627-41 & $1 \mathrm{E} \mathrm{2259+586^{c }}$ & $1 \mathrm{E} \mathrm{2259+586^{d }}$ \\
\hline Burst Energy (ergs; 25-100 keV) & $1 \times 10^{44}$ & $4 \times 10^{42}$ & $1 \times 10^{38}$ & $1 \times 10^{38}$ \\
Tail Energy (ergs; 2-10 keV) & $2 \times 10^{42}$ & $3 \times 10^{42}$ & $3 \times 10^{39}$ & $2 \times 10^{41}$ \\
Decay Index & -0.71 & -0.47 & $>-6.6$ & -0.22 \\
\hline \hline
\end{tabular}

${ }^{a}$ The following distances are assumed for conversions to energy: $15 \mathrm{kpc}$ for SGR 1900+14 (Vrba et al. 2000); $11 \mathrm{kpc}$ for SGR 1627-41 (Corbel et al. 1999); and $3.0 \mathrm{kpc}$ for 1E 2259+586 (Kothes et al. 2002).

${ }^{b}$ The values given here correspond only to those measured for the 1998 August 27 flare and its associated afterglow.

${ }^{c}$ The tail energy given here is derived from the excess persistent emission observed during the burst observations only. Because of the ambiguities in the epoch determination, an accurate decay index cannot be measured. See $\S 3.6$ for details.

$d$ The tail energy given here is derived from the excess persistent emission observed during the long time scale decay. See $§ 3.6$ for details.

The burst and persistent emission energetics and temporal decay indices for all three sources, 1E 2259+586, SGR 1900+14 and SGR 1627-41 are listed in Table 5. Clearly, there are large differences in both the decay index and the ratio of burst to persistent emission energetics among the three sources. One possibility is that the response of the persistent X-ray flux to (or when accompanied by) burst activity in SGRs and AXPs varies within the group. Alternatively (or in addition), there may be two components to the SGR flux decay similar to the AXP flux evolution and the values listed in Table 6 for the SGRs reflect a mixture of the two components which contributes to the quantitative differences with the AXP values. In fact, the flux from 
SGR $1900+14$ in the months following the end of the 40 day afterglow was enhanced relative to the pre-outburst level (Woods et al. 2001). This enhancement could be due to the persistent, low-level burst activity observed during this time interval or perhaps due to a slower flux decay component, analagous to the slow flux decay seen in $1 \mathrm{E} 2259+586$. Unfortunately, there are no reported spectral measurements of SGR $1900+14$ during this epoch to help distinguish between the two possibilities. For SGR 1627-41, the initial follow-up observation was not performed until $\sim 50$ days after the primary outburst (Woods et al. 1999a), therefore, any short-lived transient flux decay would have been missed. Thus, it appears that two flux components may possibly be present in all SGR/AXP outbursts. More rigorous follow-up spectral measurements after future outbursts are needed to show whether or not this behavior is ubiquitous.

\subsubsection{Comparison to SGR Outbursts: Pulse Properties}

SGR 1900+14 has shown one clear instance of a correlated change in the pulse profile and pulsed fraction following the burst of 1998 August 29 (Lenters et al. 2002). Here, the pulsed fraction increased to a maximum value of $\sim 20 \%$ at $\sim 200$ s following the burst, then rapidly decayed back to the pre-burst value of $\sim 12 \%$ within $10^{4} \mathrm{~s}$. During the first $\sim 100 \mathrm{~s}$, the pulse shape showed large changes relative to the pre-burst profile, and similar to the pulsed fraction recovery, recovered fully (within the errors) during the next $10^{4} \mathrm{~s}$. In $1 \mathrm{E} 2259+586$, we observe comparable changes in both the pulse shape and pulsed fraction at early times ( $\$ 6$ days) during the outburst. However, the time scale is significantly longer for $1 \mathrm{E} 2259+586$ and the pulsed fraction decreased rather than increased.

The X-ray pulse profile of SGR 1900+14 changed from a complex, multi-peaked shape before the giant flare of 1998 August 27 to a simple, nearly sinusoidal profile following the flare (Woods et al. 2001). This change has persisted for years following the outburst and has yet to recover (Göğüs et al. 2002). The constraints on any change in pulsed fraction in SGR 1900+14 following this flare are weak, as the first reported pulsed fraction measurement was made at 19 days following the flare, consistent with the pre-flare value (Woods et al. 2001). In 1E 2259+586, there was a significant change in the pulse profile in which the power in the fundamental frequency increased relative to the higher harmonics, analogous to what was observed in SGR 1900+14. The difference in pulse shape from before to during/following the burst activity was not as profound as the change seen in SGR 1900+14, however, the burst activity in 1E 2259+586 was not nearly as energetic either. Note also that the change in pulse profile of $1 \mathrm{E} 2259+586$ was transient as the pulse shape at one year following the outburst is very similar to the pre-outburst pulse profile. 


\subsubsection{Physical Interpretation}

In spite of the quantitave differences mentioned above, many of the features of the outburst in 1E 2259+586 are qualitatively very similar to those seen in SGR outbursts - specifically, the flux enhancement, spectral change, and correlated change in pulse properties. The similarities outlined above further solidify the connection between SGRs and AXPs. Combined with the similar burst characteristics (Gavriil et al. 2003), this outburst in 1E 2259+586 shows beyond any reasonable doubt that SGRs and AXPs are of the same nature, as predicted uniquely by the magnetar model.

During the rapid initial flux decay, the blackbody radius was smaller $(\sim 1 \mathrm{~km})$ than at all other times and the temperature was higher $(0.8-1.7 \mathrm{keV})$. The thermal component of the spectrum suggests the existence of a hot spot that is either cooling through its surface or is being heated by energetic particles accelerated in the magnetosphere. A localized hot spot will clearly result in a change in the emitted radiation pattern (i.e. pulse profile) even if the pulse shape is strongly modified by scattering in the magnetosphere (Thompson, Lyutikov, \& Kulkarni 2002). The reduction in pulsed fraction suggests that the heated region is offset in angle from the locations on (or above) the stellar surface that give rise to the two pulse maxima.

These observations do not allow us to distinguish convincingly between magnetospheric emission, and passive cooling of an impulsively heated crust, as the underlying source of the transient X-ray emission. The rapid flux decay could have a very different time scaling from what is observed following intermediate energy bursts in SGR 1900+14, and any change in the magnetic field of $1 \mathrm{E} 2259+586$ may have occured too gradually to generate a bright X-ray outburst. A large current density will be excited in the magnetosphere above regions of strong crustal shear, but the mechanism by which this current is damped depends on how rapidly it is excited (Thompson \& Duncan 2001). The rapid flux decay as measured is consistent with a gradually diminishing creep within a small region of the crust over a period of $\sim 10^{5} \mathrm{~s}$.

It is also worth considering whether this part of the X-ray transient is the direct aftermath of a more energetic burst. The short-lived afterglows detected after SGR bursts of intermediate energy have a simple explanation as the cooling of a pair-rich surface layer heated by a high-energy burst (Ibrahim et al. 2001), and as such are valuable probes of the burst mechanism. As estimated above, the minimum energy of such a burst is $\sim 1.5 \times 10^{41} \mathrm{ergs}$, and its duration must exceed $\sim 300 \mathrm{~s}$ if the hard X-ray flux is to remain below the limit imposed by the IPN detectors.

Is there any evidence for such extended energy release during SGR outbursts? SGR 1900+14 has, in fact, been observed to radiate the same energy over time scales ranging from $\sim 100 \mathrm{~s}$ in the declining tail of the August 27 flare (Feroci et al. 2001), down to $\sim 2 \mathrm{sec}$ in the August 29 burst that followed it. This difference in time scales was ascribed by Ibrahim et al. (2001) to a difference in fireball geometry. It should be noted at this point that the dipole magnetic field inferred from spin down is more than an order of magnitude larger for SGR 1900+14 (Kouveliotou et al. 1999) than for $1 \mathrm{E} 2259+586$. In the 'trapped fireball' model (Thompson \& Duncan 1995) this global field strength controls the rate at which a confined magnetospheric plasma can cool 
and contract: this cooling time scales as $\sim B^{-2}$ if the temperature of the escaping X-ray photons is regulated to $\gtrsim 12 \mathrm{keV}$ by the rate of photon splitting in the super-QED magnetic field (see eqs. [93] and [35] in Thompson \& Duncan 1995), and as $\sim B^{-4 / 3}$ when the photosphere remains near local thermodynamic equilibrium (see eqs [180] and [45] in Thompson \& Duncan 1995). So, there is some reason (on both observational and theoretical grounds) for taking seriously the possibility of long-duration, intermediate-energy hard X-ray outbursts from $1 \mathrm{E} 2259+586$. We note that the internal field strength of $1 \mathrm{E} 2259+586$ could be much stronger, as could the field in the region of the prompt X-ray activity (Gavriil et al. 2002).

We now comment on the relative importance of crustal heating and enhanced magnetospheric emission for the slow decay component of the outburst of $1 \mathrm{E} 2259+586$. Bulk heating of the crust of a magnetar can power an excess heat flux from its surface for a year or longer, and has been proposed as the explanation for the quasi-power-law flux decay seen in SGR 1900+14 (Lyubarsky, Eichler \& Thompson 2002) and SGR 1627-41 (Kouveliotou et al. 2003). In each case, an initial energy deposition of $10^{44} \mathrm{ergs}$ was assumed. For SGR $1900+14$, there was in fact a giant flare preceding the flux enhancement that emitted $\sim 10^{44}$ ergs in soft gamma-rays. For SGR 1627-41, the burst energy output was more than one order of magnitude less, so it was suggested that the process that generates the burst energy was much less efficient in this source (Kouveliotou et al. 2003). As discussed previously, the relative energy released in burst and excess persistent emission remains very uncertain in the case of $1 \mathrm{E} 2259+586$. Still, the excess persistent energy output in the slow decay component $\left(2 \times 10^{41} \mathrm{ergs}\right)$ was only a factor 10 lower than the output from the two SGRs, yet there was not a single burst detected with an isotropic luminosity above $\sim 1 \times 10^{39}$ ergs $\mathrm{s}^{-1}$ during this outburst. Thus, if we ascribe the slow flux decay seen in these three sources to deep heating of the crust of a magnetar, then it follows that $1 \mathrm{E} 2259+586$ is almost certainly less efficient in producing burst emission than either of the other two SGRs. Note also that the similarity in the time scales for the relaxation of the torque and the X-ray pulse profile must be coincidental if the slow decay component of the X-ray emission is powered by crustal cooling.

A small twist of the stellar crust, as is hypothesized to explain the glitch characteristics (§4.2.4), will impart a twist in the field lines that are anchored to this patch of crust. This drives a current along these twisted field lines which ultimately produces X-ray emission whose luminosity is proportional to the twist angle (Thompson et al. 2000; Thompson et al. 2002). However, following a twisting motion of the crust and external magnetic field, only a fraction $\sim 10^{-2}\left(B_{\text {dipole }} / 10^{14} \mathrm{G}\right)^{2}$ of the energy released is stored in the external non-potential magnetic field. The majority of the energy is dissipated through the deformation of the crust itself, because the shear modulus at the base of the crust corresponds to a much stronger magnetic field, $B=(4 \pi \mu)^{1 / 2} \simeq 4 \times 10^{15} \mathrm{G}$. The amplitude of the required global twist (about $\sim 10^{-2}$ radians) will only slightly change the optical depth to resonant cyclotron scattering, or the external torque through a flaring out of the external dipole field (Thompson et al. 2002). Hence, this cannot explain the large shape changes seen in the pulse profile within the first week following the glitch. It is possible, however, that the more subtle pulse profile changes seen more than one week post-glitch can be explained as a 
slight change in optical depth due to a twisting of (likely extended) field lines. The torque is more sensitive to a current localized on the most extended field lines, as is discussed in $§ 4.2 .5$.

\subsection{Rotational Evolution}

The timing data clearly show that a large spin-up glitch occurred in $1 \mathrm{E} 2259+586$ coincident with or perhaps shortly before the onset of burst activity. A portion of the frequency jump $(\sim 20 \%)$ recovered in a quasi-exponential manner within the next $\sim 60$ days. The long-term timing in the year following the glitch shows a significant reduction in the spin-down rate of this pulsar. Below, we draw comparisons between the characteristics of the $1 \mathrm{E} 2259+586$ glitch with those of other neutron stars and offer possible explanations for the observed behavior in 1E $2259+586$.

\subsubsection{Comparison with Radio Pulsars: Overall Energetics}

A conservative bound on the change in rotational energy is obtained by treating the star as a rotating solid body. For a star having moment of inertia $I=10^{45} \mathrm{~g} \mathrm{~cm}^{2}$, one infers a change in the rotational energy of $3 \times 10^{39} \mathrm{ergs}$, some two orders of magnitude smaller than the integrated excess $\mathrm{X}$-ray emission. If the glitch involves an exchange of angular momentum between a superfluid and the crust of the star, then the release of rotational energy is $\Delta E_{g} \sim(2 \pi)^{2} I_{c} \Delta \nu\left(\nu_{\mathrm{sf}}-\nu_{c}\right)$, where $\nu_{\mathrm{sf}}-\nu_{c}$ is the equilibrium lag between the rotation frequency of the superfluid and crust. Evaluating this lag using the observed $\sim 15$-d post-glitch relaxation time, one finds a value for $\Delta E_{g}$ that is smaller by a factor $10^{-3}\left(I_{\mathrm{sf}} / .02 I\right)^{-1}$. Thus the rotation of the star is manifestly not the source of energy for the transient X-ray emission.

The relaxation of elastic strains in the stellar crust is another possible source of energy. The magnitude $\psi$ of these strains could easily exceed the equilibrium rotational bulge of the star, $\left(R_{\text {equator }}-R_{\text {pole }}\right) / R_{\mathrm{NS}}=\Omega^{2} R_{\mathrm{NS}}^{3} / G M_{\mathrm{NS}}=10^{-8}(P / 7 \mathrm{~s})^{-2}$. However, the energy stored in the strained crust is $\sim 3 \times 10^{39}\left(\psi / 10^{-4}\right)^{2}$ ergs. The magnitude of the strain must, by way of comparison, be smaller than $\sim 5 \times 10^{-5}$ in the precessing pulsar PSR B1828-11 (Cutler, Ushomirsky, and Link 2003), whose spin is an order of magnitude faster than that of $1 \mathrm{E} 2259+586$. We conclude that the observed X-ray transient could be powered by the release of elastic energy

only if departure from spherical shape in the crust were comparable to that of a neutron star with $\mathrm{a} \sim 15 \mathrm{~ms}$ spin period.

The remaining possiblity is that the transient X-ray emission is powered by magnetic field decay. The energy carried by the (exterior) magnetic field of $1 \mathrm{E} 2259+586$ is comfortably larger: $8 \times 10^{44}\left(B_{\text {pole }} / 10^{14} \mathrm{G}\right)^{2}\left(R_{\mathrm{NS}} / 10 \mathrm{~km}\right)^{3}$ ergs in the simplest case of a centered dipole. This is clearly a lower bound to the total magnetic energy of the star, as it does not account for the internal (e.g. toroidal) field. The surface field (and magnetic energy) would also be significantly stronger, by a factor $\sim\left(\Delta R / 2 R_{\mathrm{NS}}\right)^{-3}$, if the dipole moment were offset from the center of the star 
(with a separation $\Delta R$ between the magnetic poles).

\subsubsection{Comparison with the AXP 1RXS J1708-4009}

It is interesting to compare the 1E $2259+586$ glitch with those reported for a different AXP, 1RXS 1708-4009. This AXP has shown two glitches, one in 1999 (Kaspi, Lackey \& Chakrabarty 2000) and one in 2001 (Kaspi \& Gavriil 2003; Dall'Osso et al. 2003). The first glitch was similar to those seen in the Vela and other radio pulsars; it showed a step $\Delta \nu / \nu=6 \times 10^{-7}$ with $Q=0$, and $\Delta \dot{\nu} / \dot{\nu} \simeq 1 \%$, such that the magnitude of the spin-down increased. The 2001 glitch, however, was markedly different, and in some ways resembled the glitch seen in $1 \mathrm{E} 2259+586$. Specifically, it showed $\Delta \nu / \nu=1.4 \times 10^{-7}$, which completely recovered exponentially on a time scale of 50 days (i.e. $Q \approx 1$ ). Whether it suffered a step in $\dot{\nu}$ depends on the interpretation of an apparent $\ddot{\nu}$ detected between glitches; this is discussed in more detail by Kaspi \& Gavriil (2003) and Dall'Osso et al. (2003). As argued by Kaspi \& Gavriil (2003), this glitch may have indicated that 1RXS 1708-4009 underwent bursting activity some time in the few week interval between monitoring observations that straddled the glitch epoch, but which went unobserved. However, no flux or pulse profile changes comparable to those seen for $1 \mathrm{E} 2259+586$ still weeks after its outburst were seen for 1RXS 1708-4009. Thus, any radiative changes would have had to decay on shorter time scales than seen in $1 \mathrm{E} 2259+586$, and in particular on shorter time scales than the exponential glitch relaxation time scale. This must be kept in mind when considering the coincidence in time scales of the $1 \mathrm{E} 2259+586$ rotational exponential decay and the average pulse profile's relaxation to its pre-outburst morphology.

\subsubsection{Comparison to $S G R s$}

From pulse frequency measurements leading up to and following the August 27 flare, it was determined that SGR $1900+14$ likely underwent rapid spin down $\left(\Delta \nu / \nu \sim-1 \times 10^{-4}\right)$ at or near the time of the flare (Woods et al. 1999b). An analysis of the pulsations during the flare itself (Palmer 2002) confirmed the existence of a timing anomaly, but only an upper limit on the time scale for the frequency change was measured ( $\$ 1$ day). For $1 \mathrm{E} 2259+586$, we detect a sudden increase in the rotation rate, as opposed to a decrease, in addition to a much smaller magnitude as that inferred for SGR 1900+14. In general, the available limits on glitches (of either sign) in SGRs are poor. This is a result of fewer and less extensive phase coherent timing solutions for these sources due to their strong timing noise (Woods et al. 2002) and weaker pulsed signals relative to most AXPs. Given the observational limitations for monitoring glitch activity in the SGRs, it is not clear whether the glitch that accompanied the outburst in $1 \mathrm{E} 2259+586$ is common to SGR outbursts.

Although there are not any lengthy phase coherent solutions bridging the boundary between 
quiescent and burst active states in SGRs to allow for identification of glitches, there are sufficient observations during and following a few outbursts to search for transient changes in spin-down rate. During the first two months following the outburst of 1E $2259+586$, the frequency derivative may have changed sign (assuming the pulse shape changes did not affect our phase measurements, see $\S 3.1)$ then rapidly switched back to spin down with a higher magnitude compared to pre-outburst. The average spin-down rate during the first two months after the glitch in 1E 2259+586 was approximately double the pre-outburst value. Similar behavior has not been seen during or following SGR outbursts. The fairly well sampled frequency histories of two SGRs $(1900+14$ and 1806-20) show that there is no direct correlation between burst activity and enhancements in the spin-down rate during or shortly following the outburst (Woods et al. 2002). In fact, the spin-down rate of SGR 1900+14 in the $\sim 40$ days following the 1998 August 27 flare was at its lowest (in magnitude) historical value (Woods et al. 1999b, 2002).

\subsubsection{Post-Glitch Relaxation}

In radio pulsars, post-glitch relaxation is almost always observed, and can be classified into two regimes: one in which the initial frequency jump relaxes exponentially relatively quickly (i.e. time scales of hours to weeks), and one in which the frequency jump does not relax, and which is generally accompanied by a permanent or very slowly (time scales of years) relaxing increase in the magnitude of the frequency derivative. Many radio pulsar glitches show both such behaviors (e.g. Shemar \& Lyne 1996, Wang et al. 2000). These are today generally interpreted in terms of vortex creep theory, in which the glitches are due to sudden unpinning of angular momentum vortices in the neutron star crustal superfluid (Anderson \& Itoh 1975). The latter, in the model, rotates faster than the crust, but in equilibrium is loosely coupled to it via pinning of vortices to crustal lattice sites. In this picture, the vortices creep outward slowly due to thermal activation (Alpar et al. 1984a,b), slowly transferring angular momentum to the crust. In equilibrium, the crust, slowed down by the external torque of magnetic braking, shares an identical spin-down rate with the superfluid, in spite of a non-zero angular velocity differential between them. Vortex creep theory identifies the two observational post-glitch relaxation regimes with different pinning regions (Alpar et al. 1989, 1993): the exponential relaxation is associated with superfluid regions with temperatures high compared to pinning energies, so that equilibrium can be established with only a small angular velocity lag. This linear regime relaxation is mathematically equivalent to the original two-component model for the crust-superfluid interaction suggested by Baym et al. (1969) which did not incorporate the more recent vortex pinning theory. When the temperature is low compared to the pinning energy, a large lag is needed to establish equilibrium; this is the non-linear regime and may be responsible for the longer-timescale relaxations of spin-down rate enhancements.

For 1E 2259+586, qualitatively, we detect both such classical glitch behaviors as well. However, quantitatively, the post-glitch relaxation of $1 \mathrm{E} 2259+586$ is very different from that 
seen in radio pulsars. Specifically, the exponential decay time is longer than what is observed in most pulsar glitches, and the $Q$ value, the fraction of the glitch that heals, is also large by pulsar standards, though neither value is extreme. What is extreme is the combination: the net effect is that the pulsar, at one month post-glitch, spins down for a couple weeks with more than double its long-term pre-outburst spin-down rate. In radio pulsars, the spin-down enhancement is typically a few percent, nearly two orders of magnitude smaller. This is because the spin-down enhancement is due to a temporarily reduced moment of inertia, as a linear component of the crustal neutron superfluid re-establishes equilibrium, and this superfluid has at most a few percent of the moment of inertia of the crust. As discussed by Kaspi et al. (2003), a spin-down enhancement of order unity would imply that half of the stellar moment of inertia was effectively decoupled from the crust following the glitch, much more than ought to be present in the crustal superfluid. Indeed the crust itself has a relatively small moment of inertia compared to the core, which is thought to be coupled to the crust on short time scales (Alpar, Langer \& Sauls 1984; Alpar \& Sauls 1988). Hence, the post-glitch relaxation of $1 \mathrm{E} 2259+586$ could imply a core decoupling. This would provide the best evidence yet for the existence of core superfluid.

However, the observed post-glitch relaxation can be understood in terms of conventional crustal superfluid if it is assumed that the superfluid/crust angular velocity lag temporarily reversed at the time of the glitch (as was noted by Alpar, Pines, \& Cheng 1990 in the case of the 1988 Christmas glitch of the Vela pulsar). The standard two-component theory has as equations of motion for the crust, of moment of inertia $I_{\mathrm{c}}$ (including everything coupled to it on short time scales), and superfluid, of moment of inertia $I_{\mathrm{sf}}$ :

$$
I_{\mathrm{c}} \dot{\Omega}_{\mathrm{c}}=\frac{I_{\mathrm{sf}}\left(\Omega_{\mathrm{sf}}-\Omega_{\mathrm{c}}\right)}{\tau}-N_{\mathrm{ex}}
$$

and

$$
I_{\mathrm{sf}} \dot{\Omega}_{\mathrm{sf}}=-\frac{I_{\mathrm{sf}}\left(\Omega_{\mathrm{sf}}-\Omega_{\mathrm{c}}\right)}{\tau},
$$

where $N_{e x}$ is the external torque on the crust and $\tau$ is the crust-superfluid energy dissipation rate. The dissipation rate can be estimated from the post-glitch exponential recovery time scale. From these equations, it follows that the change in the crustal angular frequency derivative is given by

$$
\Delta \dot{\Omega}_{\mathrm{c}}=\frac{I_{\mathrm{sf}}}{I_{\mathrm{c}}} \frac{\Delta\left(\Omega_{\mathrm{sf}}-\Omega_{\mathrm{c}}\right)}{\tau}
$$

or

$$
\Delta\left(\Omega_{\mathrm{sf}}-\Omega_{\mathrm{c}}\right)=\Delta \dot{\Omega}_{\mathrm{c}} \frac{I_{\mathrm{c}}}{I_{\mathrm{sf}}} \tau
$$

For $\tau \simeq 15$ days, $I_{\mathrm{sf}} / I_{\mathrm{c}} \simeq 0.01$ and $\Delta \dot{\Omega}_{\mathrm{c}} \simeq 2 \pi \times 10^{-14} \mathrm{rad} \mathrm{s}{ }^{-2}$, we find $\Delta\left(\Omega_{\mathrm{sf}}-\Omega_{\mathrm{c}}\right) \simeq 8 \times 10^{-6} \mathrm{rad} \mathrm{s}^{-1}$. Since $\Delta\left(\Omega_{\mathrm{sf}}-\Omega_{\mathrm{c}}\right)=\Delta \Omega_{\mathrm{sf}}-\Delta \Omega_{\mathrm{c}}$ and $\Delta \Omega_{\mathrm{c}}=3 \times 10^{-6} \mathrm{rad} \mathrm{s}^{-1}$, we have that $\Delta \Omega_{\mathrm{sf}} \simeq 5 \times 10^{-6} \mathrm{rad} \mathrm{s}^{-1}$. As the lag was initially small and $\Omega_{\mathrm{c}} \simeq 0.9 \mathrm{rad} \mathrm{s}^{-1}$, this implies the superfluid angular velocity changed by only a part in 200,000 . However, this change was about twice the observed change in the crust. Thus, at the time of the glitch, in this picture, 
the superfluid was temporarily spun down twice as much as the crust spun up. The observed enhanced spin-down post-glitch is then due to the crust transferring angular momentum back to the superfluid, as the latter attempts to arrange its vortices so as to re-establish equilibrium.

The overall activity in glitches is a measure of the moment of inertia of the internal flywheel which gives up angular momentum during glitch events (Link, Epstein \& Lattimer 1999). The activity parameter is obtained by summing over all glitches measured over a long time interval $T$. If the frequency lag between the superfluid and the star is sourced only by the external torque, then one has

$$
\frac{1}{\langle\dot{\nu}\rangle T} \sum_{i} \Delta \nu_{i} \leq \frac{I_{\mathrm{sf}}}{I}
$$

In the case of $1 \mathrm{E} 2259+586$, we estimate $\Delta \nu / \nu=4 \times 10^{-6}$ over an interval of $T=10$ yrs (the last outburst of $1 \mathrm{E} 2259+586$ being estimated to have occured 10 years earlier [Iwasawa et al. 1992]; see also $\S 4.3$ ). The resulting activity parameter is $\sim 0.1$, several times larger than what is measured in glitching radio pulsars (Link et al. 1999). Either $I_{\mathrm{sf}}$ is proportionately larger, or the spin rate of the superfluid is being reduced by some mechanism other than vortex line unpinning and thermal creep. For example, smooth deformations of the stellar crust, driven by magnetic stresses, can have the latter effect (Thompson et al. 2000).

A variety of models have been proposed to explain the origin of glitches in radio pulsars. In most models, the rotational lag between the superfluid and the crust is the principle source of free energy. The frequency lag may, as a result, decrease in magnitude during the glitch - but it is more difficult to reverse its sign. For example, Ruderman (1991) suggested that the tension of the superfluid vortices would fracture the crust, thereby allowing an outward shift in the positions of the vortices. Link and Epstein (1996) noted that any deposition of heat (of the order of $\sim 10^{41}-10^{42}$ ergs in the deep crust) would accelerate the creep rate of the pinned vortex lines, thereby causing a transient spin down of the superfluid, and a spin up of the rest of the star. Our observation of enhanced X-ray emission from 1E 2259+586 is, indeed, consistent with this level of heat deposition in the crust. But it appears that this cannot be the entire mechanism responsible for the observed glitch, because the vortex creep rate goes to zero as the spin rate of the superfluid approaches that of the crustal lattice.

In a slowly rotating neutron star, magnetic stresses can act on time scales much shorter than the external torque. Glitch-like events will result either from sudden fractures of the crust (Thompson \& Duncan 1993); or from more gradual plastic deformations during which the vortices remain pinned (Thompson et al. 2000). The lowest energy deformations of this type are torsional. Consider, for example, a twisting motion of a circular patch of the crust that is offset in azimuth from the rotation axis. Starting from a uniform distribution, more vortices are advected outward than inward (due to the curvature of the stellar surface). A twist of the order of $10^{-2}$ radians is enough to provide the required spin down of the crustal superfluid in 1E 2259+586 (eq. [12] of Thompson et al. 2000). By forcing the superfluid to rotate more slowly than it otherwise would, this mechanism also provides an explanation for the unusually large glitch activity that we inferred 
for 1E 2259+586 (see also Heyl \& Hernquist 1999).

Finally, as noted by Kaspi et al. (2003), the spin-down rate enhancement could also be due to a magnetospheric restructuring which causes the external torque to approximately double. However, given that the spin up itself is inescapably enabled by an internal transfer of angular momentum, it would have to be a suprising coincidence that the external torque change offset, to within a factor of 4-5, the internally generated frequency jump. This of course does not preclude some magnetospheric restructuring; indeed the residual pulse profile change following the initial rapid flux decay and the post-outburst infrared enhancement (Kaspi et al. 2003) suggest that likely occured. However, it seems unlikely that a magnetospheric restructuring is the cause of the enhanced spin down just post-outburst.

\subsubsection{Post-Glitch Long-Term Rotation}

Our glitch fitting clearly measured a long-term post-glitch change in the spin-down rate. Interestingly, unlike that seen in a number of radio pulsars to date, the magnitude of the spin-down torque decreased. (In the case the Crab pulsar, for example, the cumulative effect of the torque changes observed following some glitches is to increase the rate of spin-down by $0.07 \%$ over $23 \mathrm{yr}$ of observations [Lyne, et al. 1993]). If due to a superfluid effect, the conventionally observed

long-term increase in magnitude of $\dot{\Omega}_{\mathrm{c}}$ represents a reduction in the moment of inertia of the part of the neutron star that is coupled to its crust. This suggests that some fraction of the crustal superfluid is tightly pinned not creeping outward, hence decoupled from the stellar spin-down (Alpar et al. 1994), with this fraction increasing at the glitch. The opposite must therefore be true of $1 \mathrm{E} 2259+586$ in this scenario. It has alternatively been suggested that the effect is due to a secular change in the magnetic moment (Ruderman 1991); or to a change in the electromagnetic torque acting on the star, driven by a reorientation of the magnetic moment with respect to the angular velocity of the star (Link \& Epstein 1997).

We now comment on the relative merits of a change in internal vs. external torque in the case of $1 \mathrm{E} 2259+586$. The sign of the torque change is interesting, because there is independent evidence that the spin-down torque of $1 \mathrm{E} 2259+586$ has decayed over time: its characteristic age, $\tau_{c}=2 \times 10^{5} \mathrm{yrs}$, is an order of magnitude greater than that of the SNR CTB 109 (estimated radius 12 pc; Kothes et al. 2002). A change in vortex creep rate with a time scale $\tau$ much longer than $1 \mathrm{yr}$, but much less than the characteristic age $\tau_{c}=2 \times 10^{5} \mathrm{yrs}$, would appear as a permanent change in braking torque. The required moment of inertia of the superfluid component would be $I_{\text {sf }} / I=0.05\left(\tau / \tau_{c}\right)$ - comfortably below the fraction inferred for glitching radio pulsars (Link, Epstein \& Lattimer 1999) unless the creep time scale were more than a million times longer than the observed 15 day relaxation. The simplest explanation for the required change is the external torque $-\mathrm{a} \sim 2.5 \%$ reduction in the magnetic moment - can be discounted. If, for example, the external magnetic field of $1 \mathrm{E} 2259+586$ were a centered dipole, the total field energy outside the star would amount to some $10^{45} \mathrm{ergs}$, and the reduction in field energy would be a few hundred 
times larger that the excess X-ray emission we have observed.

Nonetheless, changes in external torque can result from more subtle effects. If the magnetosphere is globally twisted due to the action of internal magnetic stresses, the resulting expansion of the poloidal field lines increases the current crossing the speed of light cylinder, and therefore yields a greater torque than would exist from a simple dipolar field rotating in a vacuum (Thompson et al. 2002). Much of this effect results from the closed field lines which extend far from the star to a large radius $R_{\max }$, are anchored in a small fraction $\sim \frac{1}{2}\left(R_{\max } / R_{\mathrm{NS}}\right)^{-1}$ of its surface area, and which contain only a small fraction $\sim\left(R_{\max } / R_{\mathrm{NS}}\right)^{-3}$ of the energy in the non-potential (toroidal) field outside the star. A slight relaxation in the twist of the closed field lines is therefore consistent with the observed energetic output following the glitch, if that reconfiguration occurs close to the magnetic poles. Persistent seismic activity in a magnetar will also modulate the spin-down torque, through a coupling of crustal shear waves to the magnetosphere which in turns drives a particle wind (Thompson \& Blaes 1998; Harding, Contopoulos \& Kazanas 1999; Thompson et al. 2000). In this case, the observed change in torque corresponds to a very small reduction in the particle luminosity, by only $\sim 0.1 I \Omega \dot{\Omega} \sim 10^{31} \mathrm{erg} \mathrm{s}^{-1}$.

Finally, a change in torque could result from the excitation of precession during the glitch, followed by a permanent reorientation of the magnetic axis relative to the rotation axis of the star (Link \& Epstein 1997). That would require a precession angle of at least a few degrees, which is uncomfortably large if the non-spherical shape of the star is predominantly due to its magnetic field. The fractional difference in the sizes of the principal moments is $\sim 10^{-6}\left[\left\langle B^{2}\right\rangle /\left(10^{15} \mathrm{G}\right)^{2}\right]$ (Wasserman 2003). However, the observed energy of the X-ray transient corresponds to less than $\sim 10^{-3}$ of the total magnetic energy of the star $(\S 4.2 .1)$ and, thus, to a proportionately small change in the shape of the star.

\subsection{Implications for the Number of Active Magnetars}

As suggested by Kaspi et al. (2003), past reports of flux variability (Iwasawa et al. 1992; Baykal \& Swank 1996), pulse profile changes (Iwasawa et al. 1992), and glitch activity (Heyl \& Hernquist 1999) in 1E 2259+586 likely indicate previous episodes of burst activity in this source. The best example of an inferred outburst from 1E 2259+586 comes from a pair of Ginga observations in 1989 and 1990. Between 1989 and 1990, the pulse profile of 1E 2259+586 changed drastically, the flux increased by more than a factor 2 , and the measured frequency showed evidence of a glitch. The close similarities with the well studied outburst of 2002 June strongly suggests that a bursting episode from 1E 2259+586 preceded the 1990 Ginga observation by roughly one week. Similar reports of flux variability and timing anomalies in the AXP 1E 1048.1-5937 (e.g. Oosterbroek et al. 1998) suggests past episodes of burst activity in this source as well.

What is most intruiging about the detected and inferred outbursts in AXPs is that none has been detected with large FOV gamma-ray detectors (e.g. BATSE, Konus, Ulysses, etc.) that 
traditionally detect burst active episodes in SGRs. This shows that we are missing low intensity SGR-like outbursts from magnetar candidates in our Galaxy (i.e. the SGRs are a sensitivity limited sample).

The number of active SGRs in our Galaxy was calculated previously to be $\sim 10$ (Kouveliotou et al. 1993). A key assumption that was made in this estimation was that SGR outbursts are easily detected (i.e. there are always bright bursts within a given outburst that would trigger at least some of the large FOV gamma-ray instruments). The discovery of low-intensity SGR-like outbursts in AXPs clearly invalidates that assumption, making the estimate of 10 a lower limit only.

The future prospects for detecting weak SGR-like outbursts and addressing the question of the number of active SGRs in our Galaxy is promising. Up until 2000 June, BATSE was the most sensitive large FOV detector for SGR burst emissions. The impending Swift mission and its sensitive BAT detector will be $\sim 20$ times more sensitive than BATSE was (D. Band, private communication). Just as the RXTE PCA opened our eyes to the larger population of low-luminosity SGR bursts (e.g. Göğüş et al. 1999), perhaps Swift will reveal a previously unknown source population of dimmer SGRs.

\section{Summary}

In 2002 June, the AXP 1E 2259+586 was observed to emit more than 80 SGR-like bursts. Accompanying this outburst were several changes in the persistent X-ray emission properties of the source, many of which are similar to what has been seen in SGRs, thus further blurring the distinction between the two classes. We have quantified the observed changes using data obtained with $X M M-N e w t o n$ and $R X T E$. In particular, we found the following:

- The flux increased by more than an order of magnitude and showed two decay components. The first component decayed rapidly within the first day of the outburst while the second decayed much more slowly as a power law in time according to $t^{-0.22}$.

- The X-ray spectrum hardened during the outburst, but almost fully recovered within three days. The spectrum at 21 days past the burst activity was significantly harder than at 7 days pre-outburst, but fully consistent with historical spectral measurements of this source in quiescence.

- The phase dependence of the energy spectrum changed from before to after the outburst. One week prior to the outburst, we observe significant variablility in the photon index, but not the blackbody temperature. Three days following the outburst, the dependence of the photon index on pulse phase flattened significantly. 
- The pulse profile changed suddenly during the observation containing the burst activity where much of the power moved to the fundamental frequency. The pulse profile rapidly returned to near its pre-outburst shape within one week, and showed only very slow recovery thereafter. As with the flux and spectral changes, the recovery was not complete even after one year.

- The pulsed fraction decreased during the outburst to $\sim 15 \%$, but quickly recovered to the pre-outburst value of $\sim 23 \%$ within six days.

- 1E $2259+586$ suffered a glitch having an ordinary amplitude $\left(\Delta \nu_{\max } / \nu=(4.24 \pm 0.11) \times 10^{-6}\right)$, but a unique recovery. Approximately $19 \%$ of the glitch recovered on a time scale of $\sim 16$ days, although the recovery was not exactly exponential in form. During the recovery, the torque was enhanced relative to the pre-outburst value by a factor $\sim 2$. We detect a long-term $\sim 2 \%$ reduction in the spin-down rate following the glitch $\left(\Delta \dot{\nu}=2.18 \pm 0.25 \times 10^{-16} \mathrm{~Hz} \mathrm{~s}^{-1}\right)$.

- The measured glitch epoch preceedes the observed burst activity by $\sim 12$ hours. Given the rapid flux decay during the outburst, the true onset of this burst activity may have followed the glitch.

The cumulative properties of the outburst in $1 \mathrm{E} 2259+586$ lead us to conclude that the star suffered some major event that was extended in time and had two components, one tightly localized on the surface of the star (i.e. a fracture or a series of fractures) and the second more broadly distributed (possibly involving a smoother plastic change). This event affected both the superfluid interior and the magetosphere. The glitch points toward a disturbance within the superfluid interior while the extended flux enhancement and pulse profile change suggests an excitation of magnetospheric currents and crustal heating. Finally, we show that the lack of detection of AXP outbursts with all-sky gamma-ray detectors suggests there exists a larger population of active SGRs in our Galaxy than previously thought.

Acknowledgements - We thank P. Plucinsky (PI of the CTB 109 observations) for providing $X M M-N e w t o n$ data on 1E 2259+586. We would like to thank W. Becker, R. Epstein, M. Finger, A. Harding, C. Kouveliotou, B. Link, F. Özel, D. Psaltis, and I. Wasserman for useful discussions. We thank J. Swank and the RXTE scheduling team. We thank F. Jansen for scheduling a ToO observation with XMM-Newton. PMW acknowledges support through NASA. VMK is a Canada Research Chair, an NSERC Steacie Fellow, and a Fellow of the CIAR. Funding to VMK and FG was provided by an NSERC Discovery Grant and Steacie Supplement, NATEQ, CIAR, and NASA. CT is supported by the NSERC of Canada. JSH is supported under a Chandra Postdoctoral Fellowship Award. 


\section{REFERENCES}

Alpar, M.A., Chau, H.F., Cheng, K.S., \& Pines, D. 1993, ApJ, 409, 345

Alpar, M.A., Chau, H.F., Cheng, K.S., \& Pines, D. 1994, ApJ, 427, L29

Alpar, M.A., Cheng, K.S., \& Pines, D.A. 1989, ApJ, 346, 823

Alpar, M.A., Langer, S.A., \& Sauls, J.A. 1984, ApJ, 282, 533

Alpar, M.A., Pines, D., Anderson, P.W., \& Shaham, J. 1984a, ApJ, 276, 325

Alpar, M.A., Anderson, P.W., Pines, D., \& Shaham, J. 1984b, ApJ, 278, 791

Alpar, M.A., Pines, D., \& Cheng, K.S. 1990, Nature, 384, 707

Alpar, M.A. \& Sauls, J.A. 1988, ApJ, 327, 723

Anderson, P.W. \& Itoh, N. 1975, Nature, 256, 25

Baym, G., Pethick, C., \& Pines, D. 1969, Nature, 224, 673

Baykal, A. \& Swank, J. 1996, ApJ, 460, 470

Bildsten, L., et al. 1997, ApJS, 113, 367

Corbel, S., Chapuis, C., Dame, T.M., \& Durouchoux, P. 1999, ApJ, 526, L29

Cutler, C., Ushomirsky, G., \& Link, B. 2003, ApJ, 588, 975

Dall'Osso, S., Israel, G.L., Stella, L., Possenti, A., \& Perozzi, E. 2003, ApJ, in press

den Herder, J.W., et al. 2001, A\&A, 365, L7

Feroci, M., Hurley, K., Duncan, R.C. \& Thompson, C. 2001, ApJ, 549, 1021

Feroci, M., et al. 2003, ApJ, 596, 470

Gavriil, F. \& Kaspi, V.M. 2002, ApJ, 567, 1067

Gavriil, F. P., Kaspi, V. M., \& Woods, P. M. 2002, Nature, 419, 142

Gavriil, F. P., Kaspi, V. M., and Woods, P. M. 2003, ApJ submitted (companion paper)

Gögüus, E., Woods, P.M., Kouveliotou, C., van Paradijs, J., Briggs, M.S., Duncan, R.C., \& Thompson, C. 1999, ApJ, 526, L93

Göğüs, E., Kouveliotou, C.,, Woods, P.M., Thompson, C., Duncan, R.C. \& Briggs, M.S. 2001, ApJ, 558, 228

Gögüus, E., Kouveliotou, C., Woods, P.M., Finger, M.H., \& van der Klis, M. 2002, ApJ, 577, 929

Haberl, F., Freyberg, M., Briel, U.G., Dennerl, K., \& Zavlin, V.E. 2003, SPIE Conf. Proc.

Harding, A.K., Contopoulos, I., \& Kazanas, D. 1999, ApJ, 525, L125

Heyl, J.S. \& Hernquist, L. 1999, MNRAS, 304, L37

Ibrahim, A., Strohmayer, T.E., Woods, P.M., Kouveliotou, C., Thompson, C., Duncan, R.C., Dieters, S., van Paradijs, J. \& Finger M. 2001, ApJ, 558, 237 
Iwasawa, K., Koyama, K. \& Halpern, J.P. 1992, PASJ, 44, 9

Jansen, F., et al. 2001, A\&A, 365, L1

Jahoda, K., Swank, J.H., Giles, A.B., Stark, M.J., Strohmayer, T., Zhang, W., \& Morgan, E.H. 1996, Proc. SPIE, 2808, 59

Kaspi, V.M., Chakrabarty, D., \& Steinberger, J. 1999, ApJ, 525, L33

Kaspi, V.M., Lackey, J.R., \& Chakrabarty, D., 1999, ApJ, 537, L31

Kaspi, V.M., Gavriil, F.P., Chakrabarty, D., Lackey, J.R. \& Muno, M.P. 2001, ApJ, 558, 253

Kaspi, V.M., Gavriil, F.P., Woods, P.M., Jensen, J.B., Roberts, M.S.E., \& Chakrabarty, D. 2003, ApJ, 588, L93

Kaspi, V.M. \& Gavriil, F.P. 2003, ApJ, 596, L71

Kothes, R., Uyaniker, B., \& Aylin, Y. 2002, ApJ, 576, 169

Kouveliotou, C., et al. 1993, Nature, 362, 728

Kouveliotou, C. 2003, in Proceedings Jan van Paradijs Memorial Symposium "From X-ray Binaries to Gamma-Ray Bursts", Eds. E. van den Heuvel, L. Kaper, E. Rol, ASP Conf. Series, in press

Kouveliotou, C., et al. 2003, ApJ, 596, L79

Lenters, G., Woods, P.M., Goupell, J., Kouveliotou, C., Göğüss, E., Hurley, K., Frederiks, D., Golenetskii, S., \& Swank, J. 2003, ApJ, 587, 761

Link, B. \& Epstein, R.I. 1996, ApJ, 457, 844

Link, B. \& Epstein, R.I. 1997, ApJ, 478, L91

Link, B., Epstein, R.I., \& Lattimer, J.M. 1999, Phys. Rev. Lett., 83, 3362

Lyne, A.G., Pritchard, R.S., \& Graham-Smith, F. 1993, MNRAS, 265, 1003

Lyubarsky, E., Eichler, D., \& Thompson, C. 2002, ApJ, 580, L69

Marsden, D. \& White, N.E. 2001, ApJ, 551, L155

Mereghetti, S., Chiarlone, L., Israel, G.L., \& Stella, L. 2002, in Neutron Stars, Pulsars and Supernova Remnants, ed. H.W. Becker \& J. Trümper, Bad Honnef, in press; astro-ph/0205122

Oosterbroek, T., Parmar, A.N., Mereghetti, S., Israel, G.L. 1998, A\&A, 334, 925

Owens, A., Oosterbroek, T., \& Parmar, A.N 1997, A\&A, 324, L9

Özel, F. 2001, ApJ, 563, 276

Özel, F., Psaltis, D., \& Kaspi, V.M. 2001, ApJ, 563, 255

Palmer, D.M. 2002, in Soft Gamma Repeaters: The Rome 2001 Mini-Workshop, Eds. M. Feroci \& S. Mereghetti, Mem. S. A. It., vol 73, n. 2, pp. 578-583 
Parmar, A.N., Oosterbroek, T., Favata, F., Pightling, S., Coe, M.J., Mereghetti, S., \& Israel, G.L. 1998, A\&A, 330, 175

Patel, S.K., Kouveliotou, C., Woods, P.M., Tennant, A.F., Weisskopf, M.C., Finger, M.H., Göğüş, E., van der Klis, M., \& Belloni, T. 2001, ApJ, 563, L45

Perna, R., Heyl, J.S., Hernquist, L.E., Juett, A.M., \& Chakrabarty, D. 2001, ApJ, 557, 18

Ruderman, R. 1991, ApJ, 382, 576

Shemar, S.L. \& Lyne, A.G. 1996, MNRAS, 282, 677

Shinoda, K., Kii, T., Mitsuda, K., Nagase, F., Tanaka, Y., Makishima, K., \& Shibazaki, N. 1990, PASJ, 42, L27

Strüder, L., et al. 2001, A\&A, 365, L5

Thompson, C., \& Blaes, O. 1998, Phys. Rev. D, 57, 3219

Thompson, C., \& Duncan, R. 1993, ApJ, 408, 194

Thompson, C., \& Duncan, R. 1995, MNRAS, 275, 255

Thompson, C., \& Duncan, R. 1996, ApJ, 473, 322

Thompson, C., \& Duncan, R. 2001, ApJ, 561, 980

Thompson, C., Duncan, R., Woods, P.M., Kouveliotou, C., Finger, M.H., \& van Paradijs, J. 2000, ApJ, 543, 340

Thompson, C., Lyutikov, M., \& Kulkarni, S.R. 2002, ApJ, 574, 332

Turner, M., et al. 2001, A\&A, 365, L27

van der Klis, M. 1989, in Timing Neutron Stars, Eds. H. Ögelman \& E.P.J. van den Heuvel, Kluwer Academic Publishers, pp. 27-69

Vrba, F.J., Henden, A.A., Luginbuhl, C.B., Guetter, H.H., Hartmann, D.H. \& Klose, S. 2000, ApJ, 533, L17

Wang, N., Manchester, R.N., Pace, R.T., Bailes, M., Kaspi, V.M., Stappers, B.W., \& Lyne, A.G. 2000, MNRAS, 317, 843

Wasserman, I. 2003, MNRAS, 341, 1020

Wong, T., Backer, D.C., \& Lyne, A.G. 2001, ApJ, 548, 447

Woods, P.M., et al. 1999a, ApJ, 519, L139

Woods, P.M., et al. 1999b, ApJ, 524, L55

Woods, P.M., et al. 2000, ApJ, 535, L55

Woods, P.M., et al. 2001, ApJ, 552, 748

Woods, P.M., Kouveliotou, C., Gogus, E., Finger, M.H., Swank, J., Markwardt, C.B., Hurley, K., van der Klis, M. 2002, ApJ, 576, 381 
Woods, P.M. 2003, in Pulsars, AXPs, and SGRs Observed with BeppoSAX and other Observatories, Eds. G. Cusumano \& T. Mineo, Mem. S. A. It., vol xx, pp. yy 